\title{
FORMATION OF RADIAL PATTERNS VIA MIXED ATTRACTIVE AND REPULSIVE INTERACTIONS FOR SCHRÖDINGER SYSTEMS*
}

\author{
JAEYOUNG BYEON ${ }^{\dagger}$, YOUNGAE LEE BND ZHI-QIANG WANG $^{\ddagger}$ \\ Dedicated to Paul H. Rabinowitz on the occasion of his 80 th birthday
}

\begin{abstract}
This paper is concerned with the asymptotic behavior of least energy vector solutions for nonlinear Schrödinger systems with mixed couplings of attractive and repulsive forces. We focus here on the radially symmetric case while the general studies were already conducted in our earlier work [J. Byeon, Y. Sato, and Z.-Q. Wang, J. Math. Pures Appl. (9), 106 (2016), pp. 477-511], [J. Byeon, Y. Sato, and Z.-Q. Wang, J. Fixed Point Theory Appl., 19 (2017), pp. 559-583]. Though there is still the general phenomenon of component-wise pattern formation with co-existence of partial synchronization and segregation for positive least energy vector solutions as in [J. Byeon, Y. Sato, and Z.-Q. Wang, J. Math. Pures Appl. (9), 106 (2016), pp. 477-511], [J. Byeon, Y. Sato, and Z.-Q. Wang, J. Fixed Point Theory Appl., 19 (2017), pp. 559-583], in our case of radially symmetric domains, it turns out that the energy of synchronization part may be concentrated either on the center of the domain or on the boundary of the domain depending on the spatial dimension of the domain. This is a distinct new feature from [J. Byeon, Y. Sato, and Z.-Q. Wang, J. Math. Pures Appl. (9), 106 (2016), pp. 477-511], [J. Byeon, Y. Sato, and Z.-Q. Wang, J. Fixed Point Theory Appl., 19 (2017), pp. 559-583] due to the radially symmetric property. Our approach develops techniques of multiscale asymptotic estimates.
\end{abstract}

Key words. formation of patterns, mixed couplings, coupled Schrödinger equations

AMS subject classifications. 35J57, 35J50, 35J60, 35B40

DOI. $10.1137 / 18 \mathrm{M} 1196789$

1. Introduction. In this paper, we are concerned with the existence and asymptotic behavior of radially symmetric positive vector solutions for the following coupled nonlinear Schrödinger system:

$$
-\Delta u_{i}+\lambda_{i} u_{i}=\mu_{i} u_{i}^{3}+\sum_{j \neq i}^{3} \beta_{i j} u_{i} u_{j}^{2}, \quad u_{i}>0 \quad \text { in } \quad \Omega, \quad u_{i} \in H_{0}^{1}(\Omega) \quad(i=1,2,3) .
$$

Here $\Omega$ is the ball centered at the origin with radius $R_{0}$ in $\mathbb{R}^{n}$ for $n \leq 3$.

We continue our study on the qualitative effects of large mixed coupling on the system (1.1). More precisely, we examine the case of large $\beta_{12}>0, \beta_{13}<0, \beta_{23}<0$ when the domain is a ball. We are interested in the positive least energy vector

\footnotetext{
*Received by the editors June 26, 2018; accepted for publication (in revised form) February 8, 2019; published electronically April 30, 2019.

http://www.siam.org/journals/sima/51-2/M119678.html

Funding: The work of the first author was supported by Midcareer Researcher Program through the National Research Foundation of Korea funded by the Ministry of Science, ICT (NRF2017R1A2B4007816). The work of the second author was supported by the National Research Foundation of Korea (NRF) grant funded by the Korea government (MSIT) (NRF-2018R1C1B6003403). The work of the third author was supported by the NSFC grant 11771324 .

${ }^{\dagger}$ Department of Mathematical Sciences, KAIST, Daejeon 305-701, Republic of Korea (byeon@kaist.ac.kr).

${ }^{\ddagger}$ Department of Mathematics Education, Teachers College, Kyungpook National University, Daegu 41566, South Korea (youngaelee@knu.ac.kr).

$\S$ Corresponding author. School of Mathematics and Informatics, Fujian Normal University, Fuzhou 350117, China, and Department of Mathematics and Statistics, Utah State University, Logan, UT 84322 (zhi-qiang.wang@usu.edu).
} 
solutions of (1.1) in the class of radially symmetric functions. The existence of a least energy vector solution can be derived from the arguments in $[7,8]$. Our concern in this paper is the asymptotic behavior of the positive least energy solution in the class of radial functions when $\left|\beta_{i, j}\right|$ is large for $i \neq j$ and $\beta_{12}>0, \beta_{13}<0, \beta_{23}<0$. We will show that there is an aggregation phenomenon for the first and second components and segregation between the first two components and the third component. As $\left|\beta_{i, j}\right|$ is getting larger, the first two components are getting smaller. Even if the first two components are small, they concentrate on a sphere with a radius $r_{0} \geq 0$. We will see that $r_{0}=R_{0}$ for $n=1,2$ and $r_{0}=0$ for $n=3$, that is, the concentration behavior strongly depends on the spatial dimension $n$.

The systems considered in this paper arise when we study standing wave solutions of the time-dependent $m$-coupled Schrödinger systems

$$
\left\{\begin{array}{l}
-i \frac{\partial}{\partial t} \Phi_{j}=\Delta \Phi_{j}-V_{j}(y) \Phi_{j}+\mu_{j}\left|\Phi_{j}\right|^{2} \Phi_{j}+\Phi_{j} \sum_{i=1, i \neq j}^{m} \beta_{i j}\left|\Phi_{i}\right|^{2} \quad \text { in } \mathbb{R}^{n}, \\
\Phi_{j}=\Phi_{j}(y, t) \in \mathbb{C}, t>0, j=1, \ldots, m .
\end{array}\right.
$$

These systems of equations, also known as coupled Gross-Pitaevskii equations, have applications in Bose-Einstein condensates theory for multispecies Bose-Einstein condensates (see $[9,28])$. Physically, $\beta_{j j}$ and $\beta_{i j}(i \neq j)$ are the intraspecies and interspecies scattering lengths, respectively. The sign of the scattering length determines whether the interactions of states are repulsive or attractive. In the attractive case $\left(\beta_{i j}>0\right.$ for $\left.i \neq j\right)$ the components tend to be synchronized while in the repulsive case $\left(\beta_{i j}<0\right.$ for $\left.i \neq j\right)$ the components segregate componentwisely, leading to much more complicated behaviors of solutions. Various aspects of mathematical analysis on coupled nonlinear Schödinger equations have progressed extensively in recent years, e.g., $[1,2,3,4,6,7,8,9,10,11,14,16,17,19,20,21,22,24,26,27,30,31,32$, $33,34,35,36,37,38,39,40,41,42,43,44]$ and references therein. Most of these works have been done for the purely attractive case (i.e., all coupling constants are positive) or for the purely repulsive case (i.e., all coupling constants are negative). In the attractive case, solutions tend to go synchronization componentwise, leading to simpler structure of positive solutions (e.g., [1, 3, 4, 16, 17, 20, 21, 22, 24, 27, 41, 44] for more details). For the repulsive case, phase separation has been proved in several works causing more complicated solution structure of multiple positive solutions (e.g., $[2,9,10,11,19,35,36,37,38,39,40,42,43])$.

In terms of these previous studies, it is quite natural to consider the case of mixed couplings, i.e., there are both attractive and repulsive couplings simultaneously. Starting with an interesting case study of mixed coupling in [16], there have been results which appeared in $[7,8,16,32,33,35,36]$. In [16], Lin and Wei showed the existence of a bound state solution for a system of three equations with mixed couplings (two positive coupling and one negative coupling constants), and they demonstrated that the solution possesses component-segregation asymptotically when the couplings are small of different scales. For a general $m$ system with mixed couplings, Soave in [35] established the existence of solutions with at least $k$ positive components for every $k \leq m$. In [36], Soave and Tavares gave general conditions for the existence and symmetry result for least energy solutions with simultaneous mixed couplings on bounded domains or the whole space. In [32,33], Sato and Wang proved the existence and the asymptotic behavior of least energy solution for the three coupled systems on the bounded domain with large attractive coupling constant. Furthermore, Byeon, Sato, and Wang showed the new interesting componentwise asymptotic patter formations including co-existence of partial synchronization and segregation, when not only the 
attractive constant but also repulsive constant are large, under Dirichlet condition in [7] and Neumann condition in [8]. The current work continues the studies in this direction. As we show for the radially symmetric solutions, the asymptotic behavior is more delicate with spatial dimensions. For $n=1,2$, we see that for large mixed couplings, the third component concentrates at the origin while the other two components develop into a small synchronized boundary layer concentration. For $n=3$, the first two components also concentrate at the origin with synchronized small peaks. We expect that the energy estimation for the synchronization part in this paper would provide a motivation to study concentration behavior on higher dimensional manifolds, for example, interior spheres.

From now on, we consider a domain of ball with radius $R_{0}$,

$$
\Omega \equiv B_{R_{0}}(0)=\left\{x \in \mathbb{R}^{n}|| x \mid<R_{0}\right\} .
$$

Since we are interested in the case that two coupling constants are repulsive and one coupling constant is attractive and that $\left|\beta_{i j}\right|$ is large for $i \neq j$, we rewrite $\mu_{j}$ by $\beta_{j j}$, $\beta_{12}$ by $\alpha \beta_{12}, \beta_{13}$ by $-\beta \beta_{13}$, and $\beta_{23}$ by $-\beta \beta_{23}$, and consider the following system:

$$
\left\{\begin{array}{l}
\frac{d^{2} u_{1}}{d^{2}}+\frac{(n-1)}{r^{2}} \frac{d u_{1}}{d r}-\lambda_{1} u_{1}+\beta_{11}\left(u_{1}\right)^{3}+\alpha \beta_{12} u_{1}\left(u_{2}\right)^{2}-\beta \beta_{13} u_{1}\left(u_{3}\right)^{2}=0 \\
\frac{d^{2} u_{2}}{d r^{2}}+\frac{(n-1)}{r} \frac{d u_{2}}{d r}-\lambda_{2} u_{2}+\alpha \beta_{21}\left(u_{1}\right)^{2} u_{2}+\beta_{22}\left(u_{2}\right)^{3}-\beta \beta_{23} u_{2}\left(u_{3}\right)^{2}=0, \\
\frac{d^{2} u_{3}}{d r^{2}}+\frac{(n-1)}{d r} \frac{d u_{3}}{d r}-\lambda_{3} u_{3}-\beta \beta_{31}\left(u_{1}\right)^{2} u_{3}-\beta \beta_{32}\left(u_{2}\right)^{2} u_{3}+\beta_{33}\left(u_{3}\right)^{3}=0 \\
u_{i}(r)>0 \text { for } r<R_{0} \\
u_{1}\left(R_{0}\right)=u_{2}\left(R_{0}\right)=u_{3}\left(R_{0}\right)=0,
\end{array}\right.
$$

where $\beta_{i j}=\beta_{j i}>0$ for $1 \leq i, j \leq 3, \alpha, \beta>0$. In this paper we need a control of multiscale convergence rates for $\alpha, \beta \rightarrow \infty$. We will use the following notation: for $a, b \in \mathbb{R}$,

$$
\alpha^{a} \# \beta^{b} \rightarrow \infty \text { if and only if } \alpha, \beta \rightarrow \infty \text { with } \lim _{\alpha, \beta \rightarrow \infty} \frac{\alpha^{a}}{\beta^{b}}=\infty
$$

When we say $\alpha^{a} \# \beta^{b}$ is large, it means that all three quantities $\alpha, \beta, \frac{\alpha^{a}}{\beta^{b}}$ are large. To state our results we introduce some notation and limiting problems. Let us define

$$
\mathbf{H}_{r}(\Omega) \equiv\left(H_{0, r}^{1}(\Omega)\right)^{3}, \quad \text { where } H_{0, r}^{1}(\Omega)=\left\{u \in H_{0}^{1}(\Omega) \mid u(x)=u(|x|) \text { for all } x \in \Omega\right\} .
$$

For $p \geq 1$ and $\lambda>0$, we use the following notation:

$$
|u|_{p, \Omega}^{p}=\int_{\Omega}|u|^{p} d x, \quad\|u\|_{\lambda, \Omega}^{2}=|\nabla u|_{2, \Omega}^{2}+\lambda|u|_{2, \Omega}^{2}, \quad\|\vec{u}\|=\left(\sum_{i=1}^{3}\left\|u_{i}\right\|_{\lambda_{i}, \Omega}^{2}\right)^{\frac{1}{2}} .
$$

We define the energy functional for the system (1.3),

$$
\begin{aligned}
I(\vec{u})= & \sum_{i=1}^{3}\left(\frac{1}{2}|| u_{i}||_{\lambda_{i}, \Omega}^{2}-\frac{1}{4} \beta_{i i}\left|u_{i}\right|_{4, \Omega}^{4}\right) \\
& -\frac{1}{2}\left(\alpha \beta_{12}\left|u_{1} u_{2}\right|_{2, \Omega}^{2}-\beta \beta_{13}\left|u_{1} u_{3}\right|_{2, \Omega}^{2}-\beta \beta_{23}\left|u_{2} u_{3}\right|_{2, \Omega}^{2}\right)
\end{aligned}
$$

for $\vec{u}=\left(u_{1}, u_{2}, u_{3}\right) \in \mathbf{H}_{r}(\Omega)$. Then critical points of $I$ correspond to solutions of the system (1.3). A solution $\vec{u}=\left(u_{1}, u_{2}, u_{3}\right)$ is called a vector solution if $u_{i} \neq 0$ for each 
$i=1,2,3$. A positive least energy vector solution of (1.3) is a vector solution whose components are all positive and whose energy is the least among all vector solutions.

It is well known that the following equation has a positive least energy solution:

$$
\Delta u-\lambda_{3} u+\beta_{33}(u)^{3}=0, \quad u>0 \text { in } \Omega, \quad u=0 \text { on } \partial \Omega .
$$

We denote the least energy level for (1.4) by $L$. It is well known that for a ball $\Omega=B_{R}(0)$, the positive solution of (1.4) is unique [15]. We will show that the third component of the least energy solution of (1.3) converges to the positive solution of a limit problem (1.4). On the other hand, the first and second components have a different type of convergence and the corresponding limit problem depends on the space dimension $n$ as follows (here, we will use $t$ as the one dimensional variable for the limiting problem).

(1) $n=1,2$ (concentration on the boundary). Given the positive least energy solution $U_{3}$ of (1.4), we consider the following problem:

$$
\left\{\begin{array}{cc}
\frac{d^{2} v_{1}}{d t^{2}}-\beta_{13}\left(\frac{\partial U_{3}}{\partial r}\left(R_{0}\right)\right)^{2} t^{2} v_{1}+\beta_{12} v_{1}\left(v_{2}\right)^{2}=0, v_{1}>0 & \text { in } \mathbb{R}_{+}^{1}, \\
\frac{d^{2} v_{2}}{d t^{2}}-\beta_{23}\left(\frac{\partial U_{3}}{\partial r}\left(R_{0}\right)\right)^{2} t^{2} v_{2}+\beta_{21}\left(v_{1}\right)^{2} v_{2}=0, v_{2}>0 & \text { in } \mathbb{R}_{+}^{1}, \\
v_{i}(0)=0, \lim _{|x| \rightarrow \infty} v_{i}(x)=0, i=1,2 . &
\end{array}\right.
$$

Here we note that the term $\frac{(n-1)}{t} \frac{d}{d t}$ does not appear in the equation above since (1.5) would be a limiting equation for (3.29), and the first derivative term for the solution in (3.29) would vanish as $\beta \rightarrow \infty$.

The corresponding energy functional $B_{U_{3}}$ is defined by

$$
\begin{aligned}
& B_{U_{3}}\left(v_{1}, v_{2}\right) \\
& \equiv \frac{1}{2} \int_{\mathbb{R}_{+}^{1}}\left|\frac{d v_{1}}{d t}\right|^{2}+\left|\frac{d v_{2}}{d t}\right|^{2}+t^{2}\left(\frac{\partial U_{3}}{\partial r}\left(R_{0}\right)\right)^{2}\left(\beta_{13} v_{1}^{2}+\beta_{23} v_{2}^{2}\right)-\beta_{12} v_{1}^{2} v_{2}^{2} d t
\end{aligned}
$$

for $\left(v_{1}, v_{2}\right) \in H_{b}^{1}$, where $H_{b}^{1}$ is defined by the completion of $C_{0}^{\infty}\left(\mathbb{R}_{+}^{1}\right)$ with respect to the norm

$$
\left\|\left(v_{1}, v_{2}\right)\right\|_{b}^{2} \equiv \int_{\mathbb{R}_{+}^{1}}\left|\frac{d v_{1}}{d t}\right|^{2}+\left|\frac{d v_{2}}{d t}\right|^{2}+t^{2}\left(\beta_{13} v_{1}^{2}+\beta_{23} v_{2}^{2}\right) d t
$$

We note that for $\breve{v}=\left(v_{1}, v_{2}\right)$

$$
B_{U_{3}}^{\prime}(\breve{v})(\breve{v})=\int_{\mathbb{R}_{+}^{1}}\left|\frac{d v_{1}}{d t}\right|^{2}+\left|\frac{d v_{2}}{d t}\right|^{2}+t^{2}\left(\frac{\partial U_{3}}{\partial r}\left(R_{0}\right)\right)^{2}\left(\beta_{13} v_{1}^{2}+\beta_{23} v_{2}^{2}\right)-2 \beta_{12} v_{1}^{2} v_{2}^{2} d t .
$$

Then, we consider the following minimization problem:

$$
M_{b}=M_{b}\left(U_{3}\right) \equiv \inf \left\{B_{U_{3}}(\breve{v}) \mid B_{U_{3}}^{\prime}(\breve{v})(\breve{v})=0, \breve{v} \in H_{b}^{1} \backslash\{(0,0)\}\right\} .
$$

In view of [7, Proposition 4], we see that there exists a minimizer $\left(v_{1, b}, v_{2, b}\right)$ of $(1.7)$ which is a least energy solution of (1.5). Moreover, there exist constants $c, C>0$ satisfying

$$
0<v_{i, b}(t) \leq C e^{-c t^{2}} \text { for any } t>0, i=1,2
$$

Copyright (c) by SIAM. Unauthorized reproduction of this article is prohibited. 
By the scaling $v_{i, b}(t)=\sqrt{\left|\frac{\partial U_{3}}{\partial r}\left(R_{0}\right)\right|} v_{i}^{0}\left(\sqrt{\left|\frac{\partial U_{3}}{\partial r}\left(R_{0}\right)\right|} t\right), i=1,2$, we see that

$$
M_{b}=M_{b}\left(U_{3}\right)=\left|\frac{\partial U_{3}}{\partial r}\left(R_{0}\right)\right|^{\frac{3}{2}} M_{b}^{0},
$$

where $M_{b}^{0}$ is the energy of a least energy solution $\left(v_{1, b}^{0}, v_{2, b}^{0}\right) \in H_{b}^{1}\left(\mathbb{R}_{+}^{1}\right)$ of the following normalized problem:

$$
\left\{\begin{array}{cc}
\frac{d^{2} v_{1, b}^{0}}{d t^{2}}-\beta_{13} t^{2} v_{1, b}^{0}+\beta_{12} v_{1, b}^{0}\left(v_{2, b}^{0}\right)^{2}=0, v_{1, b}^{0}>0 & \text { in } \mathbb{R}_{+}^{1}, \\
\frac{d^{2} v_{2, b}^{0}}{d t^{2}}-\beta_{23} t^{2} v_{2, b}^{0}+\beta_{21}\left(v_{1, b}^{0}\right)^{2} v_{2, b}^{0}=0, v_{2, b}^{0}>0 & \text { in } \mathbb{R}_{+}^{1}, \\
v_{i, b}^{0}(0)=0, \lim _{|x| \rightarrow \infty} v_{i, b}^{0}(x)=0, i=1,2 . &
\end{array}\right.
$$

(2) $n=3$ (concentration on the center). For the positive least energy solution $U_{3}$ of (1.4), we consider the following problem:

$$
\left\{\begin{array}{r}
\Delta v_{1}-\beta_{13}\left(U_{3}(0)\right)^{2} v_{1}+\beta_{12} v_{1}\left(v_{2}\right)^{2}=0, v_{1}>0 \\
\Delta v_{2}-\beta_{23}\left(U_{3}(0)\right)^{2} v_{2}+\beta_{21}\left(v_{1}\right)^{2} v_{2}=0, v_{2}>0 \\
v_{1}, v_{2} \in H_{0, r}^{1}\left(\mathbb{R}^{n}\right)
\end{array}\right.
$$

The corresponding energy functional $C_{U_{3}}$ is defined by

$$
C_{U_{3}}\left(v_{1}, v_{2}\right) \equiv \frac{1}{2} \int_{\mathbb{R}^{n}}\left(\left|\nabla v_{1}\right|^{2}+\left|\nabla v_{2}\right|^{2}+\left(U_{3}(0)\right)^{2}\left(\beta_{13} v_{1}^{2}+\beta_{23} v_{2}^{2}\right)-\beta_{12} v_{1}^{2} v_{2}^{2}\right) d y .
$$

We also note that for $\breve{v}=\left(v_{1}, v_{2}\right) \in H_{c}^{1} \equiv\left(H_{0, r}^{1}\left(\mathbb{R}^{n}\right)\right)^{2}$,

$$
C_{U_{3}}^{\prime}(\breve{v})(\breve{v}) \equiv \int_{\mathbb{R}^{n}}\left(\left|\nabla v_{1}\right|^{2}+\left|\nabla v_{2}\right|^{2}+\left(U_{3}(0)\right)^{2}\left(\beta_{13} v_{1}^{2}+\beta_{23} v_{2}^{2}\right)-2 \beta_{12} v_{1}^{2} v_{2}^{2}\right) d y .
$$

We consider the following minimization problem:

$$
M_{c}=M_{c}\left(U_{3}\right) \equiv \inf \left\{C_{U_{3}}(\breve{v}) \mid C_{U_{3}}^{\prime}(\breve{v})(\breve{v})=0, \quad \breve{v} \in H_{c}^{1} \backslash\{(0,0)\}\right\} .
$$

In view of [8, Proposition 4], there exists a minimizer $\left(v_{1, c}, v_{2, c}\right)$ of $(1.12)$ which is a least energy solution of (1.11) and radially symmetric up to a translation. Moreover, there are constants $c, C>0$ satisfying

$$
0<v_{i, c}(y) \leq C e^{-c|y|} \text { for any } y \in \mathbb{R}^{n}, i=1,2 .
$$

By the scaling $v_{i, c}(y)=U_{3}(0) v_{i, c}^{0}\left(U_{3}(0) y\right), i=1,2$, we note that

$$
M_{c}\left(U_{3}\right)=\left(U_{3}(0)\right)^{4-n} M_{c}^{0},
$$

where $M_{c}^{0}$ is the energy of a least energy solution of the following normalized problem:

$$
\left\{\begin{array}{r}
\Delta v_{1, c}^{0}-\beta_{13} v_{1, c}^{0}+\beta_{12} v_{1, c}^{0}\left(v_{2, c}^{0}\right)^{2}=0, v_{1, c}^{0}>0 \\
\Delta v_{2, c}^{0}-\beta_{23} v_{2, c}^{0}+\beta_{21}\left(v_{1, c}^{0}\right)^{2} v_{2, c}^{0}=0, v_{2, c}^{0}>0 \\
v_{1, c}^{0}, v_{2, c}^{0} \in H_{0, r}^{1}\left(\mathbb{R}^{n}\right) .
\end{array}\right.
$$

From now on, for any domain $O \subset \mathbb{R}^{n}$, any function $u \in H_{0}^{1}(O)$ will also be regarded as an element in $H^{1}\left(\mathbb{R}^{n}\right)$ by the zero extension $u$ on $\mathbb{R}^{n} \backslash O$.

Copyright (c) by SIAM. Unauthorized reproduction of this article is prohibited. 
Throughout this paper, we often use $t$ as the one dimensional variable for the limiting problem, $x=r \theta$, where $r=|x|$ and $\theta=\frac{x}{|x|}$, as the original variable in $\Omega$, and $y, s=|y|$ as the scaled variables such that

$$
\left\{\begin{array}{l}
y=\beta^{\frac{1}{4}}\left(R_{0}-r\right) \theta, s=|y| \text { if } n=1,2 \\
y=\sqrt{\beta} x, s=|y|
\end{array}\right.
$$

Our main result is the following theorem.

TheOREm 1. We assume that $\Omega=B_{R_{0}}(0)$. We take any $\delta_{1}, \delta_{2} \geq \frac{1}{4}$ for $n=1,2$ and any $\delta_{3}>0$ for $n=3$. Then the following holds.

(a) There exists a constant $\alpha_{0}>0$, independent of $\beta>0$ such that for any $\alpha \geq \alpha_{0}$, $\beta>0$, the system (1.3) has a least energy vector solution $\vec{u}^{\alpha, \beta}=\left(u_{1}^{\alpha, \beta}, u_{2}^{\alpha, \beta}, u_{3}^{\alpha, \beta}\right)$ satisfying

$$
\begin{gathered}
\lim _{\alpha \rightarrow \infty} I\left(\vec{u}^{\alpha, \beta}\right)=L \text { uniformly for } \beta>0 \text { and } \\
\lim _{\alpha \rightarrow \infty}\left\|\vec{u}^{\alpha, \beta}-\left(0,0, U_{3}\right)\right\|=0 \quad \text { uniformly for } \beta>0,
\end{gathered}
$$

where $L$ is the least energy level of (1.4), and $U_{3}$ is the unique least energy solution of (1.4).

$$
I\left(\vec{u}^{\alpha, \beta}\right)=\left\{\begin{array}{l}
L+\frac{\beta^{\frac{3}{4}}}{\alpha}\left(M_{b}\left|S^{n-1}\right| R_{0}^{n-1}+o(1)\right) \text { as } \alpha \# \beta^{1+\delta_{n}} \rightarrow \infty \text { if } n=1,2, \\
L+\frac{\beta^{\frac{1}{2}}}{\alpha}\left(M_{c}+o(1)\right) \text { as } \alpha \# \beta^{1+\delta_{n}} \rightarrow \infty \text { if } n=3,
\end{array}\right.
$$

where $\left|S^{n-1}\right|$ is the area of the unit sphere.

(c) For $n=1,2$ and $u_{i}^{\alpha, \beta}(x)=u_{i}^{\alpha, \beta}(|x|)$, we define

$v_{i, b}^{\alpha, \beta}(|y|) \equiv \frac{\sqrt{\alpha}}{\beta^{\frac{1}{4}}} u_{i}^{\alpha, \beta}\left(R_{0}-\frac{|y|}{\beta^{\frac{1}{4}}}\right), i=1,2$, for $|y| \in \Omega_{\beta, b} \equiv\left\{|y| \in \mathbb{R}|0 \leq| y \mid \leq \beta^{\frac{1}{4}} R_{0}\right\}$.

Then there is a positive least energy vector solution $\left(v_{1, b}, v_{2, b}\right)$ of (1.5) such that $\left(v_{1, b}^{\alpha, \beta}, v_{2, b}^{\alpha, \beta}\right) \rightarrow\left(v_{1, b}, v_{2, b}\right)$ in $\left[H^{1}(\mathbb{R})\right]^{2}$, up to a subsequence, as $\alpha \# \beta^{1+\delta_{n}} \rightarrow \infty$. Moreover, there are constants $c, C, D>0$, independent of large $\alpha \# \beta^{1+\delta_{n}}$ such that for $i=1,2$,

$$
D \frac{\beta^{\frac{1}{4}}}{\sqrt{\alpha}} \leq \max _{\Omega} u_{i}^{\alpha, \beta}, \quad u_{i}^{\alpha, \beta}(x) \leq C \frac{\beta^{\frac{1}{4}}}{\sqrt{\alpha}} \exp \left(-c\left(\beta^{\frac{1}{4}}\left(R_{0}-|x|\right)\right)\right), \quad 0 \leq|x| \leq R_{0} .
$$

(d) For $n=3$, we define

$$
v_{i, c}^{\alpha, \beta}(y)=\sqrt{\frac{\alpha}{\beta}} u_{i}^{\alpha, \beta}\left(\frac{y}{\sqrt{\beta}}\right), \quad i=1,2, \quad \text { for } \quad y \in \Omega_{\beta, c} \equiv\left\{y \in \mathbb{R}^{n}|| y \mid \leq \sqrt{\beta} R_{0}\right\} .
$$

Then there is a positive least energy vector solution $\left(v_{1, c}, v_{2, c}\right)$ of (1.11) such that $\left(v_{1, c}^{\alpha, \beta}, v_{2, c}^{\alpha, \beta}\right) \rightarrow\left(v_{1, c}, v_{2, c}\right) \quad$ in $\left[H^{1}\left(\mathbb{R}^{n}\right)\right]^{2}$, up to a subsequence, as $\alpha \# \beta^{1+\delta_{n}} \rightarrow \infty$.

Moreover, there are constants $c, C, D>0$, independent of large $\alpha \# \beta^{1+\delta_{n}}$ such that for $i=1,2$,

$$
D \sqrt{\frac{\beta}{\alpha}} \leq \max _{\Omega} u_{i}^{\alpha, \beta}, \quad u_{i}^{\alpha, \beta}(x) \leq C \sqrt{\frac{\beta}{\alpha}} \exp (-c \sqrt{\beta}|x|), \quad 0 \leq|x|<R_{0} .
$$

Copyright (C) by SIAM. Unauthorized reproduction of this article is prohibited. 
Theorem 1 indicates that the concentration region of the least energy vector solution for the synchronization part depends on the dimension of the domain. As we saw in [7], without the radial constraint, a concentration point of the first and second components moves to the boundary even though the Dirichlet boundary condition is imposed. In the radially symmetric case, if $n=3$, the expense of the concentration of the first and second components on the sphere is much higher than the concentration at the center of a ball. In order to obtain the asymptotic behavior of the synchronization part, we divide the domain into two parts and use the different norms on each regions.

The paper is organized as follows. In section 2 , we review some preliminaries to establish the existence results for (1.3). In section 3, we introduce a combined norm to obtain energy estimates depending on the dimension of the domain, and complete the proof of Theorem 1.

2. Preliminary. For $\vec{u}=\left(u_{1}, u_{2}, u_{3}\right) \in \mathbf{H}_{r}(\Omega)$, we define

$$
\begin{aligned}
l\left(u_{1}, u_{2}, u_{3}\right) \equiv & \frac{1}{2} \sum_{i=1}^{3}\left(\left|\nabla u_{i}\right|^{2}+\lambda_{i}\left(u_{i}\right)^{2}-\frac{1}{2} \beta_{i i}\left|u_{i}\right|^{4}\right) \\
& -\frac{1}{2}\left(\alpha \beta_{12}\left|u_{1} u_{2}\right|^{2}-\beta \beta_{13}\left|u_{1} u_{3}\right|^{2}-\beta \beta_{23}\left|u_{2} u_{3}\right|^{2}\right) .
\end{aligned}
$$

Recall the energy functional $I(\vec{u})=\int_{\Omega} l\left(u_{1}, u_{2}, u_{2}\right) d x$ for $\vec{u}=\left(u_{1}, u_{2}, u_{3}\right) \in \mathbf{H}_{r}(\Omega)$, and define

$\mathcal{D}_{\alpha, \beta} \equiv\left\{\vec{u} \in \mathbf{H}_{r}(\Omega) \mid I^{\prime}(\vec{u})\left(u_{1}, u_{2}, 0\right)=0, I^{\prime}(\vec{u})\left(0,0, u_{3}\right)=0,\left(u_{1}, u_{2}\right) \neq(0,0), u_{3} \neq 0\right\}$,

$\widetilde{\mathcal{D}}_{\alpha, \beta} \equiv\left\{\vec{u} \in \mathbf{H}_{r}(\Omega) \mid I^{\prime}(\vec{u})\left(u_{1}, u_{2}, 0\right) \leq 0, I^{\prime}(\vec{u})\left(0,0, u_{3}\right) \leq 0,\left(u_{1}, u_{2}\right) \neq(0,0), u_{3} \neq 0\right\}$,

$\widehat{\mathcal{D}}_{\alpha, \beta} \equiv\left\{\vec{u} \in \mathbf{H}_{r}(\Omega) \mid \operatorname{det} A_{\alpha, \beta}(\vec{u})>0\right\}$,

where the matrix $A_{\alpha, \beta}(\vec{u})$ is given by

$$
\left[\begin{array}{cc}
\beta_{11}\left|u_{1}\right|_{4, \Omega}^{4}+\beta_{22}\left|u_{2}\right|_{4, \Omega}^{4}+2 \alpha \beta_{12}\left|u_{1} u_{2}\right|_{2, \Omega}^{2} & -\left(\beta \beta_{13}\left|u_{1} u_{3}\right|_{2, \Omega}^{2}+\beta \beta_{23}\left|u_{2} u_{3}\right|_{2, \Omega}^{2}\right) \\
-\left(\beta \beta_{13}\left|u_{1} u_{3}\right|_{2, \Omega}^{2}+\beta \beta_{23}\left|u_{2} u_{3}\right|_{2, \Omega}^{2}\right) & \beta_{33}\left|u_{3}\right|_{4, \Omega}^{4}
\end{array}\right] .
$$

Lemma 2 (see [7, Lemma 5 and Remark 6]). (i) For any $\vec{u} \in \widetilde{\mathcal{D}}_{\alpha, \beta} \cup \widehat{\mathcal{D}}_{\alpha, \beta}$, there exists a unique $\left(s_{\alpha, \beta}(\vec{u}), t_{\alpha, \beta}(\vec{u})\right) \in(0,1] \times(0,1]$ such that

$$
\begin{aligned}
\left(s_{\alpha, \beta}(\vec{u}) u_{1}, s_{\alpha, \beta}(\vec{u}) u_{2}, t_{\alpha, \beta}(\vec{u}) u_{3}\right) & \in \mathcal{D}_{\alpha, \beta}, \\
I\left(s_{\alpha, \beta}(\vec{u}) u_{1}, s_{\alpha, \beta}(\vec{u}) u_{2}, t_{\alpha, \beta}(\vec{u}) u_{3}\right) & =\max _{s, t>0} I\left(s u_{1}, s u_{2}, t u_{3}\right) .
\end{aligned}
$$

(ii) $I\left(s_{\alpha, \beta}(\vec{u}) u_{1}, s_{\alpha, \beta}(\vec{u}) u_{2}, t_{\alpha, \beta}(\vec{u}) u_{3}\right)=\frac{H(\vec{u})}{4 G(\vec{u})}$, where

$$
\begin{aligned}
G(\vec{u})= & \left(\beta_{11}\left|u_{1}\right|_{4, \Omega}^{4}+\beta_{22}\left|u_{2}\right|_{4, \Omega}^{4}+2 \alpha \beta_{12}\left|u_{1} u_{2}\right|_{2, \Omega}^{2}\right) \beta_{33}\left|u_{3}\right|_{4, \Omega}^{4} \\
& -\left(\beta \beta_{13}\left|u_{1} u_{3}\right|_{2, \Omega}^{2}+\beta \beta_{23}\left|u_{2} u_{3}\right|_{2, \Omega}^{2}\right)^{2} \\
H(\vec{u})= & \beta_{33}\left|u_{3}\right|_{4, \Omega}^{4}\left(\left\|u_{1}\right\|_{\lambda_{1}, \Omega}^{2}+\left\|u_{2}\right\|_{\lambda_{2}, \Omega}^{2}\right)^{2} \\
& +2\left(\beta \beta_{13}\left|u_{1} u_{3}\right|_{2, \Omega}^{2}+\beta \beta_{23}\left|u_{2} u_{3}\right|_{2, \Omega}^{2}\right)\left(\left\|u_{1}\right\|_{\lambda_{1}, \Omega}^{2}+\left\|u_{2}\right\|_{\lambda_{2}, \Omega}^{2}\right)\left\|u_{3}\right\|_{\lambda_{3}, \Omega}^{2} \\
& +\left(\beta_{11}\left|u_{1}\right|_{4, \Omega}^{4}+\beta_{22}\left|u_{2}\right|_{4, \Omega}^{4}+2 \alpha \beta_{12}\left|u_{1} u_{2}\right|_{2, \Omega}^{2}\right)\left\|u_{3}\right\|_{\lambda_{3}, \Omega}^{4} .
\end{aligned}
$$

We consider a minimization problem

$$
c_{\alpha, \beta}=\inf _{\vec{u} \in \mathcal{D}_{\alpha, \beta}} I(\vec{u})=\inf _{\vec{u} \in \mathcal{D}_{\alpha, \beta}} \frac{1}{4}\left(\left\|u_{1}\right\|_{\lambda_{1}, \Omega}^{2}+\left\|u_{2}\right\|_{\lambda_{2}, \Omega}^{2}+\left\|u_{3}\right\|_{\lambda_{3}, \Omega}^{2}\right) .
$$

Copyright $@$ by SIAM. Unauthorized reproduction of this article is prohibited. 
With the aid of $[29,5]$, we argue as in [7] to have the following proposition.

Proposition 3 (see [7, Propositions 8-12]). The minimum $c_{\alpha, \beta}$ is achieved by an element $\vec{u}^{\alpha, \beta}=\left(u_{1}^{\alpha, \beta}, u_{2}^{\alpha, \beta}, u_{3}^{\alpha, \beta}\right) \in \mathcal{D}_{\alpha, \beta}$ which satisfies (1.3). Moreover, the following hold uniformly for $\beta>0$ :

(i) $u_{1}^{\alpha, \beta}, u_{2}^{\alpha, \beta}, u_{3}^{\alpha, \beta}>0$ in $\Omega$ for large $\alpha>0$.

(ii) the set $\left\{\left|u_{3}^{\alpha, \beta}\right|_{\infty, \Omega}\right\}_{\alpha \geq 1, \beta>0}$ is bounded.

(iii) $\lim _{\alpha \rightarrow \infty}\left\|\vec{u}^{\alpha, \beta}-\left(0,0, U_{3}\right)\right\|=0$, where $U_{3}$ is the least energy solution of (1.4).

(iv) $\lim _{\alpha \rightarrow \infty} c_{\alpha, \beta}=L$, where $L$ is the least energy level of (1.4).

(v) $\lim _{\alpha \rightarrow \infty}\left\|u_{1}^{\alpha, \beta}\right\|_{\lambda_{1}, \Omega}^{2}=\lim _{\alpha \rightarrow \infty}\left\|u_{2}^{\alpha, \beta}\right\|_{\lambda_{2}, \Omega}^{2}=0$.

(vi) $\lim _{\alpha \rightarrow \infty} \beta \int_{\Omega}\left(u_{1}^{\alpha, \beta}\right)^{2}\left(u_{3}^{\alpha, \beta}\right)^{2}+\left(u_{2}^{\alpha, \beta}\right)^{2}\left(u_{3}^{\alpha, \beta}\right)^{2} d x=\lim _{\alpha \rightarrow \infty} \alpha \int_{\Omega}\left(u_{1}^{\alpha, \beta}\right)^{2}\left(u_{2}^{\alpha, \beta}\right)^{2} d x=$ 0 .

Proof. The proof follows from the arguments in [7], and we omit the details. Indeed, the minimum $c_{\alpha, \beta}$ is achieved by an element $\vec{u}^{\alpha, \beta}=\left(u_{1}^{\alpha, \beta}, u_{2}^{\alpha, \beta}, u_{3}^{\alpha, \beta}\right) \in \mathcal{D}_{\alpha, \beta}$ which satisfies (1.3) by the same arguments in [7, Proposition 8]. Moreover, (iv)-(vi) are obtained from the arguments in [7, Proposition 9]. By using the arguments in [7, Proposition 10], (i) can be proved. Finally, (ii) and (iii) can be obtained from the arguments in [7, Proposition 11] and [7, Proposition 12], respectively.

3. A refined convergence by a renormalization. We recall $\vec{u}^{\alpha, \beta}=\left(u_{1}^{\alpha, \beta}\right.$, $\left.u_{2}^{\alpha, \beta}, u_{3}^{\alpha, \beta}\right)$ as a minimizer of $c_{\alpha, \beta}$. In this section, we renormalize $\left(u_{1}^{\alpha, \beta}, u_{2}^{\alpha, \beta}\right)$ so that the renormalized solution converges to a least energy solution of the elliptic system discussed in section 1. After analyzing the refined convergence, we will prove Theorem 1 at the end.

\subsection{Basic energy estimates.}

Proposition 4. $c_{\alpha, \beta}$ has the following upper estimate:

$$
\begin{aligned}
c_{\alpha, \beta} & \leq \min \left\{L+\frac{\beta^{\frac{3}{4}}}{\alpha}\left(M_{b}\left|S^{n-1}\right| R_{0}^{n-1}+o(1)\right), L+\frac{\beta^{2-\frac{n}{2}}}{\alpha}\left(M_{c}+o(1)\right)\right\} \\
& =\left\{\begin{array}{l}
L+\frac{\beta^{\frac{3}{4}}}{\alpha}\left(M_{b}\left|S^{n-1}\right| R_{0}^{n-1}+o(1)\right) \text { as } \alpha \# \beta^{\frac{3}{4}} \rightarrow \infty \text { if } n=1,2, \\
L+\frac{\beta^{\frac{1}{2}}}{\alpha}\left(M_{c}+o(1)\right) \text { as } \alpha \# \beta^{\frac{1}{2}} \rightarrow \infty \text { if } n=3,
\end{array}\right.
\end{aligned}
$$

where $L$ is the least energy level of (1.4), and $M_{b}$ and $M_{c}$ are defined in (1.7) and (1.12), respectively.

Proof. We choose a cut-off function $\chi \in C^{2}\left(\mathbb{R}^{1},[0,1]\right)$ such that $0 \leq \chi \leq 1$ and

$$
\chi(t)= \begin{cases}1 & \text { if }|t| \leq \frac{1}{4} \\ 0 & \text { if }|t| \geq \frac{1}{2}\end{cases}
$$

We consider the following cases depending on the possible location of the concentration part for the first and second components:

Case 1: Concentration on the boundary. We take the least energy solution $U_{3}$ for (1.4) and a least energy solution $\left(v_{1, b}, v_{2, b}\right)$ for (1.5). We note that

$$
4 L=\left\|U_{3}\right\|_{\lambda_{3}, \Omega}^{2}=\beta_{33}\left|U_{3}\right|_{4, \Omega}^{4},
$$




$$
\begin{aligned}
4 M_{b}\left(U_{3}\right) & =\left|\nabla v_{1, b}\right|_{2, \mathbb{R}_{+}^{1}}^{2}+\left|\nabla v_{2, b}\right|_{2, \mathbb{R}_{+}^{1}}^{2}+\int_{\mathbb{R}_{+}^{1}}\left|\frac{\partial U_{3}}{\partial r}\left(R_{0}\right)\right|^{2} t^{2}\left(\beta_{13}\left(v_{1, b}\right)^{2}+\beta_{23}\left(v_{2, b}\right)^{2}\right) d t \\
& =2 \beta_{12}\left|v_{1, b} v_{2, b}\right|_{2, \mathbb{R}_{+}^{1}}^{2} .
\end{aligned}
$$

Now, for $x \in \Omega$, we define

$$
\begin{aligned}
& \left(w_{1, b}^{\alpha, \beta}(x), w_{2, b}^{\alpha, \beta}(x), w_{3}(x)\right) \\
& \equiv\left(\frac{\beta^{\frac{1}{4}}}{\sqrt{\alpha}} v_{1, b}\left(\beta^{\frac{1}{4}}\left(R_{0}-|x|\right)\right) \chi\left(\beta^{\frac{1}{12}}\left(1-\frac{|x|}{R_{0}}\right)\right),\right. \\
& \left.\frac{\beta^{\frac{1}{4}}}{\sqrt{\alpha}} v_{2, b}\left(\beta^{\frac{1}{4}}\left(R_{0}-|x|\right)\right) \chi\left(\beta^{\frac{1}{12}}\left(1-\frac{|x|}{R_{0}}\right)\right), U_{3}(x)\right) .
\end{aligned}
$$

Then, $\left(w_{1, b}^{\alpha, \beta}, w_{2, b}^{\alpha, \beta}, w_{3}\right) \in \mathbf{H}_{r}(\Omega)$. We denote $x=r \theta \in \Omega$, where $r=|x|$ and $\theta=\frac{x}{|x|}$. By (1.8) and a change of variables $y=\beta^{\frac{1}{4}}\left(R_{0}-r\right) \theta$ and $s=|y|$, it holds that for each $i=1,2$,

$$
\begin{aligned}
& \alpha \beta_{12}\left|w_{1, b}^{\alpha, \beta} w_{2, b}^{\alpha, \beta}\right|_{2, \Omega}^{2}=\frac{\beta^{\frac{3}{4}}}{\alpha}\left\{\beta_{12}\left|v_{1, b} v_{2, b}\right|_{2, \mathbb{R}_{+}^{1}}^{2}+o_{\beta}(1)\right\}\left|S^{n-1}\right| R_{0}^{n-1} \\
& \beta \beta_{i 3}\left|w_{i, b}^{\alpha, \beta} w_{3}\right|_{2, \Omega}^{2}=\frac{\beta^{\frac{3}{4}}}{\alpha}\left\{\beta_{i 3} \int_{\mathbb{R}_{+}^{1}}\left|\frac{\partial U_{3}}{\partial r}\left(R_{0}\right)\right|^{2} s^{2}\left(v_{i, b}\right)^{2} d s+o_{\beta}(1)\right\}\left|S^{n-1}\right| R_{0}^{n-1},
\end{aligned}
$$

where $o_{\beta}(1) \rightarrow 0$ as $\beta \rightarrow \infty$. We prove only (3.8) since (3.5)-(3.7) can be proved in a similar way. Setting $y=\beta^{\frac{1}{4}}\left(R_{0}-r\right) \theta$ and $s=|y|$, we see that $d s=-\beta^{\frac{1}{4}} d r$ and $r^{n-1}$ $=\left(R_{0}-\beta^{-\frac{1}{4}} s\right)^{n-1}$. Thus it follows that

$$
\begin{aligned}
& \beta \beta_{i 3}\left|w_{i, b}^{\alpha, \beta} w_{3}\right|_{2, \Omega}^{2} \\
& =\frac{\beta^{\frac{3}{2}}}{\alpha} \beta_{i 3} \int_{0}^{R_{0}}\left\{v_{i, b}\left(\beta^{\frac{1}{4}}\left(R_{0}-r\right)\right) \chi\left(\beta^{\frac{1}{12}}\left(1-\frac{r}{R_{0}}\right)\right) U_{3}(r)\right\}^{2} r^{n-1} d r\left|S^{n-1}\right| \\
& =\frac{\beta^{\frac{3}{2}-\frac{1}{4}}}{\alpha} \beta_{i 3} \int_{0}^{\beta^{\frac{1}{6}} \frac{R_{0}}{2}}\left\{v_{i, b}(s) \chi\left(\frac{s}{\beta^{\frac{1}{6}} R_{0}}\right) U_{3}\left(R_{0}-\beta^{-\frac{1}{4}} s\right)\right\}^{2}\left(R_{0}-\beta^{-\frac{1}{4}} s\right)^{n-1} d s\left|S^{n-1}\right| .
\end{aligned}
$$

We see from the fact $U_{3}\left(R_{0}\right)=0$ and the mean value theorem that for $s \in\left[0, \frac{\beta^{\frac{1}{6}} R_{0}}{2}\right]$,

$$
U_{3}\left(R_{0}-\beta^{-\frac{1}{4}} s\right)=U_{3}\left(R_{0}-\beta^{-\frac{1}{4}} s\right)-U_{3}\left(R_{0}\right)=-\frac{d U_{3}}{d r}\left(R_{0}\right) \beta^{-\frac{1}{4}} s+O\left(\beta^{-\frac{1}{2}} s^{2}\right)
$$

From the exponential decay of $v_{1, b}, v_{2, b}$ in (1.8), we see that (3.9) and (3.10) imply (3.8).

Copyright $@$ by SIAM. Unauthorized reproduction of this article is prohibited. 
From (3.4), (3.5), (3.7), and (3.8), it follows that

$$
\begin{aligned}
\sum_{i=1,2}\left(\left\|w_{i, b}^{\alpha, \beta}\right\|_{\lambda_{i}, \Omega}^{2}+\beta \beta_{i 3}\left|w_{i, b}^{\alpha, \beta} w_{3}\right|_{2, \Omega}^{2}\right) & =\frac{\beta^{\frac{3}{4}}}{\alpha}\left(4 M_{b}+o_{\beta}(1)\right)\left|S^{n-1}\right| R_{0}^{n-1}, \\
2 \alpha \beta_{12}\left|w_{1, b}^{\alpha, \beta} w_{2, b}^{\alpha, \beta}\right|_{2, \Omega}^{2} & =\frac{\beta^{\frac{3}{4}}}{\alpha}\left(4 M_{b}+o_{\beta}(1)\right)\left|S^{n-1}\right| R_{0}^{n-1} .
\end{aligned}
$$

From (3.3), (3.11), (3.12), $\vec{w}^{\alpha, \beta} \equiv\left(w_{1, b}^{\alpha, \beta}, w_{2, b}^{\alpha, \beta}, w_{3}\right) \in \widehat{\mathcal{D}}_{\alpha, \beta}$ as $\alpha \# \beta^{\frac{3}{4}} \rightarrow \infty$. Thus from Lemma 2(i), there exists a unique $(s, t) \in \mathbb{R}^{2}$ such that $\left(s w_{1, b}^{\alpha, \beta}, s w_{2, b}^{\alpha, \beta}, t w_{3}\right) \in \mathcal{D}_{\alpha, \beta}$. By the expression in Lemma 2(ii) for $I\left(s w_{1, b}^{\alpha, \beta}, s w_{2, b}^{\alpha, \beta}, t w_{3}\right)$, we deduce that

$$
I\left(s w_{1, b}^{\alpha, \beta}, s w_{2, b}^{\alpha, \beta}, t w_{3}\right)=\frac{\left\|w_{3}\right\|_{\lambda_{3}, \Omega}^{4}}{4 \beta_{33}\left|w_{3}\right|_{4, \Omega}^{4}}+\frac{K\left(\vec{w}^{\alpha, \beta}\right)}{4 \beta_{33}\left|w_{3}\right|_{4, \Omega}^{4} G\left(\vec{w}^{\alpha, \beta}\right)},
$$

where

$$
\begin{aligned}
& K(\vec{w})=\beta_{33}^{2}\left|w_{3}\right|_{4, \Omega}^{8}\left(\left\|w_{1}\right\|_{\lambda_{1}, \Omega}^{2}+\left\|w_{2}\right\|_{\lambda_{2}, \Omega}^{2}\right)^{2} \\
& \quad+2 \beta_{33}\left|w_{3}\right|_{4, \Omega}^{4}\left(\beta \beta_{13}\left|w_{1} w_{3}\right|_{2, \Omega}^{2}+\beta \beta_{23}\left|w_{2} w_{3}\right|_{2, \Omega}^{2}\right)\left(\left\|w_{1}\right\|_{\lambda_{1}, \Omega}^{2}+\left\|w_{2}\right\|_{\lambda_{2}, \Omega}^{2}\right)\left\|w_{3}\right\|_{\lambda_{3}, \Omega}^{2} \\
& \quad+\left(\beta \beta_{13}\left|w_{1} w_{3}\right|_{2, \Omega}^{2}+\beta \beta_{23}\left|w_{2} w_{3}\right|_{2, \Omega}^{2}\right)^{2}\left\|w_{3}\right\|_{\lambda_{3}, \Omega}^{4}
\end{aligned}
$$

and

$(3.15)$

$$
\begin{aligned}
G(\vec{w})=G\left(w_{1}, w_{2}, w_{3}\right)= & \left(\beta_{11}\left|w_{1}\right|_{4, \Omega}^{4}+\beta_{22}\left|w_{2}\right|_{4, \Omega}^{4}+2 \alpha \beta_{12}\left|w_{1} w_{2}\right|_{2, \Omega}^{2}\right) \beta_{33}\left|w_{3}\right|_{4, \Omega}^{4} \\
& -\left(\beta \beta_{13}\left|w_{1} w_{3}\right|_{2, \Omega}^{2}+\beta \beta_{23}\left|w_{2} w_{3}\right|_{2, \Omega}^{2}\right)^{2} .
\end{aligned}
$$

Now, by using (3.3), (3.13) is simplified to

$$
\begin{aligned}
& I\left(s w_{1, b}^{\alpha, \beta}, s w_{2, b}^{\alpha, \beta}, t w_{3}\right) \\
& =L+\frac{L\left(\left\|w_{1, b}^{\alpha, \beta}\right\|_{\lambda_{1}, \Omega}^{2}+\left\|w_{2, b}^{\alpha, \beta}\right\|_{\lambda_{2}, \Omega}^{2}+\beta \beta_{13}\left|w_{1, b}^{\alpha, \beta} w_{3}\right|_{2, \Omega}^{2}+\beta \beta_{23}\left|w_{2, b}^{\alpha, \beta} w_{3}\right|_{2, \Omega}^{2}\right)^{2}}{G\left(\vec{w}^{\alpha, \beta}\right)} .
\end{aligned}
$$

From (3.6), (3.11), and (3.12),

$$
G\left(\vec{w}^{\alpha, \beta}\right)=\frac{\beta^{\frac{3}{4}}}{\alpha}\left(16 M_{b} L\left|S^{n-1}\right| R_{0}^{n-1}+o(1)\right) \quad \text { as } \quad \alpha \# \beta^{\frac{3}{4}} \rightarrow \infty .
$$

Thus, from (3.11) and (3.17), it follows that as $\alpha \# \beta^{\frac{3}{4}} \rightarrow \infty$,

$$
c_{\alpha, \beta} \leq I\left(s w_{1, b}^{\alpha, \beta}, s w_{2, b}^{\alpha, \beta}, t w_{3}\right)=L+\frac{\beta^{\frac{3}{4}}}{\alpha}\left(M_{b}\left|S^{n-1}\right| R_{0}^{n-1}+o(1)\right) .
$$

Since a least energy solution $U_{3}$ of (1.4) is unique, we obtain one of the upper estimates for (3.1).

Case 2: Concentration on the center. We take the least energy solution $U_{3}$ for (1.4) and a least energy solution $\left(v_{1, c}, v_{2, c}\right)$ for (1.11). We note that

$$
\begin{gathered}
4 L=|| U_{3} \|_{\lambda_{3}, \Omega}^{2}=\beta_{33}\left|U_{3}\right|_{4, \Omega}^{4} \\
4 M_{c}\left(U_{3}\right)=\left|\nabla v_{1, c}\right|_{2, \mathbb{R}^{n}}^{2}+\left|\nabla v_{2, c}\right|_{2, \mathbb{R}^{n}}^{2}+\left(U_{3}(0)\right)^{2}\left\{\beta_{13}\left|v_{1, c}\right|_{2, \mathbb{R}^{n}}^{2}+\beta_{23}\left|v_{2, c}\right|_{2, \mathbb{R}^{n}}^{2}\right\} \\
=2 \beta_{12}\left|v_{1, c} v_{2, c}\right|_{2, \mathbb{R}^{n}}^{2}
\end{gathered}
$$

Copyright $@$ by SIAM. Unauthorized reproduction of this article is prohibited. 
Now, for $x \in \Omega$, we define

$$
\begin{aligned}
& \left(w_{1, c}^{\alpha, \beta}(x), w_{2, c}^{\alpha, \beta}(x), w_{3}(x)\right) \\
& \equiv\left(\sqrt{\frac{\beta}{\alpha}} v_{1, c}(\sqrt{\beta} x) \chi\left(\beta^{\frac{1}{4}} R_{0}^{-1}|x|\right), \sqrt{\frac{\beta}{\alpha}} v_{2, c}(\sqrt{\beta} x) \chi\left(\beta^{\frac{1}{4}} R_{0}^{-1}|x|\right), U_{3}(x)\right) .
\end{aligned}
$$

We have $\left(w_{1, c}^{\alpha, \beta}, w_{2, c}^{\alpha, \beta}, w_{3}\right) \in \mathbf{H}_{r}(\Omega)$. By (1.13) and a change of variables $x=\beta^{-\frac{1}{2}} y$, it holds that for each $i=1,2$,

$$
\begin{aligned}
\left\|w_{i, c}^{\alpha, \beta}\right\|_{\lambda_{i}, \Omega}^{2} & =\frac{\beta^{2-\frac{n}{2}}}{\alpha}\left\{\left|\nabla v_{i, c}\right|_{2, \mathbb{R}^{n}}^{2}+o_{\beta}(1)\right\}, \\
\left|w_{i, c}^{\alpha, \beta}\right|_{4, \Omega}^{4} & =\frac{\beta^{2-\frac{n}{2}}}{\alpha^{2}}\left\{\left|v_{i, c}\right|_{4, \mathbb{R}^{n}}^{4}+o_{\beta}(1)\right\}, \\
\alpha \beta_{12}\left|w_{1, c}^{\alpha, \beta} w_{2, c}^{\alpha, \beta}\right|_{2, \Omega}^{2}= & \frac{\beta^{2-\frac{n}{2}}}{\alpha}\left\{\beta_{12}\left|v_{1, c} v_{2, c}\right|_{2, \mathbb{R}^{n}}^{2}+o_{\beta}(1)\right\}, \\
\beta \beta_{i 3}\left|w_{i, c}^{\alpha, \beta} w_{3}\right|_{2, \Omega}^{2}= & \frac{\beta^{2-\frac{n}{2}}}{\alpha}\left\{\beta_{i 3}\left(U_{3}(0)\right)^{2}\left|v_{i, c}\right|_{2, \mathbb{R}^{n}}^{2}+o_{\beta}(1)\right\},
\end{aligned}
$$

where $o_{\beta}(1) \rightarrow 0$ as $\beta \rightarrow \infty$. From (3.20), (3.21), (3.23), and (3.24), it follows that

$$
\begin{aligned}
\sum_{i=1,2}\left(\left\|w_{i, c}^{\alpha, \beta}\right\|_{\lambda_{i}, \Omega}^{2}+\beta \beta_{i 3}\left|w_{i, c}^{\alpha, \beta} w_{3}\right|_{2, \Omega}^{2}\right) & =\frac{\beta^{2-\frac{n}{2}}}{\alpha}\left(4 M_{c}\left(U_{3}\right)+o_{\beta}(1)\right) \\
2 \alpha \beta_{12}\left|w_{1, c}^{\alpha, \beta} w_{2, c}^{\alpha, \beta}\right|_{2, \Omega}^{2} & =\frac{\beta^{2-\frac{n}{2}}}{\alpha}\left(4 M_{c}\left(U_{3}\right)+o_{\beta}(1)\right) .
\end{aligned}
$$

By the similar arguments with Case 1 above, it follows that as $\alpha \# \beta^{2-\frac{n}{2}} \rightarrow \infty$,

$$
c_{\alpha, \beta} \leq L+\frac{\beta^{2-\frac{n}{2}}}{\alpha}\left(M_{c}+o(1)\right)
$$

By comparing (3.18) and (3.27), we complete the proof of Proposition 4.

3.2. A renormalization and basic estimates. As we will use a multiscale renormalization we introduce some notation here. We will consider two different scalings according to the scaled regions: the neighborhood of the boundary or the neighborhood of the center. In order to distinguish these different scalings and scaled regions, we will use the subscript "b" for the notation related to the scalings on the neighborhood of the boundary, and the subscript "c" for the notation related to the scalings on the neighborhood of the center.

The scaling on the neighborhood of the boundary. Let

$u_{1}^{\alpha, \beta}(x)=\frac{\beta^{\frac{1}{4}}}{\sqrt{\alpha}} v_{1, b}^{\alpha, \beta}\left(\beta^{\frac{1}{4}}\left(R_{0}-|x|\right)\right), u_{2}^{\alpha, \beta}(x)=\frac{\beta^{\frac{1}{4}}}{\sqrt{\alpha}} v_{2, b}^{\alpha, \beta}\left(\beta^{\frac{1}{4}}\left(R_{0}-|x|\right)\right), u_{3}^{\alpha, \beta}(x)=v_{3}^{\alpha, \beta}(x)$.

We denote $x=r \theta \in \Omega$, where $r=|x|$ and $\theta=\frac{x}{|x|}$. By letting $y=\beta^{\frac{1}{4}}\left(R_{0}-r\right) \theta$, $s=|y|$, and $\Omega_{b}^{\beta}=\left\{s \in \mathbb{R} \mid 0 \leq s \leq \frac{\beta^{\frac{1}{4}} R_{0}}{2}\right\}$, we see that $\vec{v}^{\alpha, \beta} \equiv\left(v_{1, b}^{\alpha, \beta}, v_{2, b}^{\alpha, \beta}, v_{3}^{\alpha, \beta}\right)$ 
satisfies

(3.29)

$$
\left\{\begin{array}{l}
\frac{d^{2} v_{1, b}^{\alpha, \beta}}{d s^{2}}-\frac{(n-1) \beta^{-\frac{1}{4}}}{R_{0}-\beta^{-\frac{1}{4}}} \frac{d v_{1, b}^{\alpha, \beta}}{d s}-\frac{\lambda_{1}}{\sqrt{\beta}} v_{1, b}^{\alpha, \beta}+\frac{\beta_{11}}{\alpha}\left(v_{1, b}^{\alpha, \beta}\right)^{3}+\beta_{12} v_{1, b}^{\alpha, \beta}\left(v_{2, b}^{\alpha, \beta}\right)^{2} \\
=\sqrt{\beta} \beta_{13} v_{1, b}^{\alpha, \beta}\left(v_{3}^{\alpha, \beta}\left(R_{0}-\beta^{-\frac{1}{4}} s\right)\right)^{2} \text { in } \Omega_{b}^{\beta}, \\
\frac{d^{2} v_{2, b}^{\alpha, \beta}}{d s^{2}}-\frac{(n-1) \beta^{-\frac{1}{4}}}{R_{0}-\beta^{-\frac{1}{4}}} \frac{d v_{2, b}^{\alpha, \beta}}{d s}-\frac{\lambda_{2}}{\sqrt{\beta}} v_{2, b}^{\alpha, \beta}+\beta_{21}\left(v_{1, b}^{\alpha, \beta}\right)^{2} v_{2, b}^{\alpha, \beta}+\frac{\beta_{22}}{\alpha}\left(v_{2, b}^{\alpha, \beta}\right)^{3} \\
=\sqrt{\beta} \beta_{23} v_{2, b}^{\alpha, \beta}\left(v_{3}^{\alpha, \beta}\left(R_{0}-\beta^{-\frac{1}{4}} s\right)\right)^{2} \text { in } \Omega_{b}^{\beta}, \\
\Delta v_{3}^{\alpha, \beta}-\lambda_{3} v_{3}^{\alpha, \beta}-\left(\sum_{i=1}^{2} \beta_{3 i}\left(v_{i, b}^{\alpha, \beta}\left(\beta^{\frac{1}{4}}\left(R_{0}-|x|\right)\right)\right)^{2}\right) \frac{\beta^{\frac{3}{2}}}{\alpha} v_{3}^{\alpha, \beta}+\beta_{33}\left(v_{3}^{\alpha, \beta}\right)^{3}=0 \text { in } \Omega .
\end{array}\right.
$$

The scaling on the neighborhood of the center. Let

$$
u_{1}^{\alpha, \beta}(x)=\sqrt{\frac{\beta}{\alpha}} v_{1, c}^{\alpha, \beta}(\sqrt{\beta} x), \quad u_{2}^{\alpha, \beta}(x)=\sqrt{\frac{\beta}{\alpha}} v_{2, c}^{\alpha, \beta}(\sqrt{\beta} x), \quad u_{3}^{\alpha, \beta}(x)=v_{3}^{\alpha, \beta}(x) .
$$

By letting $y=\beta^{\frac{1}{2}} x$ and $\Omega_{c}^{\beta}=\left\{y \in \mathbb{R}^{n}|| y \mid \leq \frac{\sqrt{\beta} R_{0}}{2}\right\}$, we see that $\vec{v}^{\alpha, \beta} \equiv$ $\left(v_{1, c}^{\alpha, \beta}, v_{2, c}^{\alpha, \beta}, v_{3}^{\alpha, \beta}\right)$ satisfies

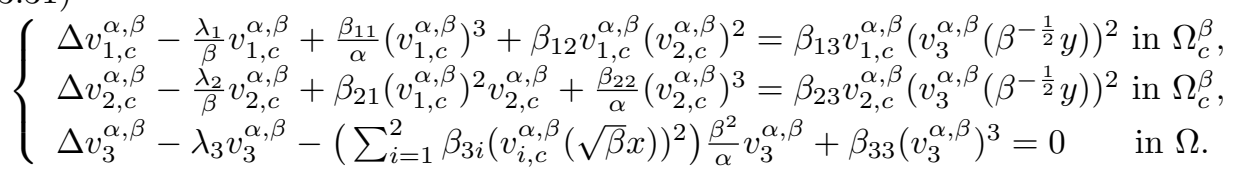

Here we denote

$$
s=|y|, \quad \Delta=\frac{d^{2}}{d s^{2}}+\frac{(n-1)}{s} \frac{d}{d s}, \quad \text { and } \quad|\nabla \phi|_{2, \Omega_{c}^{\beta}}^{2}=\left|S^{n-1}\right| \int_{0}^{\frac{\sqrt{\beta} R_{0}}{2}}\left|\frac{d \phi}{d s}\right|^{2} s^{n-1} d s
$$

Now we have the following result.

Copyright $\odot$ by SIAM. Unauthorized reproduction of this article is prohibited. 
Proposition 5. It holds that

$$
\begin{aligned}
& \frac{\beta^{2-\frac{n}{2}}}{\alpha}\left\{\frac{\beta_{11}}{\alpha}\left|v_{1, c}^{\alpha, \beta}\right|_{4, \Omega_{c}^{\beta}}^{4}+\frac{\beta_{22}}{\alpha}\left|v_{2, c}^{\alpha, \beta}\right|_{4, \Omega_{c}^{\beta}}^{4}+2 \beta_{12}\left|v_{1, c}^{\alpha, \beta} v_{2, c}^{\alpha, \beta}\right|_{2, \Omega_{c}^{\beta}}^{2}\right\} \\
& +\frac{\beta^{\frac{3}{4}}\left|S^{n-1}\right|}{\alpha}\left\{\frac{\beta_{11}}{\alpha}\left|v_{1, b}^{\alpha, \beta}\left(R_{0}-\beta^{-\frac{1}{4}}|y|\right)^{\frac{n-1}{4}}\right|_{4, \Omega_{b}^{\beta}}^{4}+\frac{\beta_{22}}{\alpha}\left|v_{2, b}^{\alpha, \beta}\left(R_{0}-\beta^{-\frac{1}{4}}|y|\right)^{\frac{n-1}{4}}\right|_{4, \Omega_{b}^{\beta}}^{4}\right. \\
& \left.+2 \beta_{12}\left|v_{1, b}^{\alpha, \beta} v_{2, b}^{\alpha, \beta}\left(R_{0}-\beta^{-\frac{1}{4}}|y|\right)^{\frac{n-1}{2}}\right|_{2, \Omega_{b}^{\beta}}^{2}\right\} \\
& =\frac{\beta^{2-\frac{n}{2}}}{\alpha}\left\{\sum_{i=1}^{2}\left(\left|\nabla v_{i, c}^{\alpha, \beta}\right|_{2, \Omega_{c}^{\beta}}^{2}+\frac{\lambda_{i}}{\beta}\left|v_{i, c}^{\alpha, \beta}\right|_{2, \Omega_{c}^{\beta}}^{2}+\beta_{i 3} \int_{\Omega_{c}^{\beta}}\left(v_{3}^{\alpha, \beta}\left(\beta^{-\frac{1}{2}} y\right)\right)^{2}\left(v_{i, c}^{\alpha, \beta}\right)^{2} d y\right)\right\} \\
& +\frac{\beta^{\frac{3}{4}}\left|S^{n-1}\right|}{\alpha}\left\{\sum_{i=1}^{2}\left(\left|\nabla v_{i, b}^{\alpha, \beta}\left(R_{0}-\beta^{-\frac{1}{4}}|y|\right)^{\frac{n-1}{2}}\right|_{2, \Omega_{b}^{\beta}}^{2}+\left.\frac{\lambda_{i}}{\sqrt{\beta}} v_{i, b}^{\alpha, \beta}\left(R_{0}-\beta^{-\frac{1}{4}} y\right)^{\frac{n-1}{2}}\right|_{2, \Omega_{b}^{\beta}} ^{2}\right)\right. \\
& \left.+\sum_{i=1}^{2} \beta_{i 3} \int \sqrt{\beta}\left(v_{3}^{\alpha, \beta}\left(R_{0}-\beta^{-\frac{1}{4}}|y|\right)\right)^{2}\left(v_{i, b}^{\alpha, \beta}\right)^{2}\left(R_{0}-\beta^{-\frac{1}{4}}|y|\right)^{n-1} d y\right\} \\
& \leq \min \left\{\frac{\beta^{\frac{3}{4}}}{\alpha}\left(4 M_{b}\left|S^{n-1}\right| R_{0}^{n-1}+o(1)\right), \frac{\beta^{2-\frac{n}{2}}}{\alpha}\left(4 M_{c}+o(1)\right)\right\} \\
& =\left\{\begin{array}{c}
\frac{\beta^{\frac{3}{4}}}{\alpha}\left(4 M_{b}\left|S^{n-1}\right| R_{0}^{n-1}+o(1)\right) \quad \text { as } \alpha \# \beta^{\frac{3}{4}} \rightarrow \infty \text { if } n=1,2, \\
\frac{\beta^{\frac{1}{2}}}{\alpha}\left(4 M_{c}+o(1)\right) \quad \text { as } \alpha \# \beta^{\frac{1}{2}} \rightarrow \infty \quad i f \quad n=3 .
\end{array}\right.
\end{aligned}
$$

Proof. In view of [7, Proposition 14], we have the following estimation:

$$
\begin{aligned}
& \beta_{11}\left|u_{1}^{\alpha, \beta}\right|_{4, \Omega}^{4}+\beta_{22}\left|u_{2}^{\alpha, \beta}\right|_{4, \Omega}^{4}+2 \alpha \beta_{12}\left|u_{1}^{\alpha, \beta} u_{2}^{\alpha, \beta}\right|_{2, \Omega}^{2} \\
& =\left\|u_{1}^{\alpha, \beta}\right\|_{\lambda_{1}, \Omega}^{2}+\left\|u_{2}^{\alpha, \beta}\right\|_{\lambda_{2}, \Omega}^{2}+\beta \beta_{13}\left|u_{1}^{\alpha, \beta} u_{3}^{\alpha, \beta}\right|_{2, \Omega}^{2}+\beta \beta_{23}\left|u_{2}^{\alpha, \beta} u_{3}^{\alpha, \beta}\right|_{2, \Omega}^{2} \\
& \leq \min \left\{\frac{\beta^{\frac{3}{4}}}{\alpha}\left(4 M_{b}\left|S^{n-1}\right| R_{0}^{n-1}+o(1)\right), \frac{\beta^{2-\frac{n}{2}}}{\alpha}\left(4 M_{c}+o(1)\right)\right\} \\
& =\left\{\begin{array}{l}
\frac{\beta^{\frac{3}{4}}}{\alpha}\left(4 M_{b}\left|S^{n-1}\right| R_{0}^{n-1}+o(1)\right) \text { as } \alpha \# \beta^{\frac{3}{4}} \rightarrow \infty \text { if } n=1,2, \\
\frac{\beta^{\frac{1}{2}}}{\alpha}\left(4 M_{c}+o(1)\right) \text { as } \alpha \# \beta^{\frac{1}{2}} \rightarrow \infty \text { if } n=3 .
\end{array}\right.
\end{aligned}
$$

The proof of Proposition 5 comes from (3.33) by taking a change of variables.

Proposition 6. For any $R \in\left(0, R_{0}\right)$, there exists a constant $C>0$ such that

$$
\begin{array}{r}
\left|u_{1}^{\alpha, \beta}\right|_{\infty, \Omega}+\left|u_{2}^{\alpha, \beta}\right|_{\infty, \Omega} \leq C \frac{\beta^{\frac{3}{8}}}{\sqrt{\alpha}} \quad \text { as } \alpha \# \beta^{\frac{3}{4}} \rightarrow \infty \quad \text { if } n=1 ; \\
\left.\left|u_{1}^{\alpha, \beta}\right|_{\infty,\{x \in \Omega|| x \mid \geq R\}}+\left|u_{2}^{\alpha, \beta}\right|_{\infty,\{x \in \Omega \mid}|x| \geq R\right\} \leq C \frac{\beta^{\frac{3}{8}}}{\sqrt{\alpha}} \quad \text { as } \alpha \# \beta^{\frac{3}{4}} \rightarrow \infty \quad \text { if } n=2 ; \\
\left|u_{1}^{\alpha, \beta}\right|_{\infty, \Omega}+\left|u_{2}^{\alpha, \beta}\right|_{\infty, \Omega} \leq C \sqrt{\frac{\beta}{\alpha}} \quad \text { as } \alpha \# \beta^{\frac{1}{2}} \rightarrow \infty \quad \text { if } n=3 .
\end{array}
$$

Proof. For $n=1$, there exists $C_{1}>0$, independent of $\beta>0$, such that for $i=1,2,\left|u_{i}^{\alpha, \beta}\right|_{\infty, \Omega} \leq C_{1}\left\|u_{i}^{\alpha, \beta}\right\|_{\lambda_{i}, \Omega}$. Then, (3.33) implies that there exists some $C_{2}>0$, independent of $\beta>0$, such that for large $\alpha \# \beta^{\frac{3}{4}}$,

$$
\left|u_{i}^{\alpha, \beta}\right|_{\infty, \Omega} \leq C_{2}\left(\frac{\beta^{\frac{3}{4}}}{\alpha}\right)^{\frac{1}{2}}=C_{2} \frac{\beta^{\frac{3}{8}}}{\sqrt{\alpha}}, \quad i=1,2 .
$$

Copyright (c) by SIAM. Unauthorized reproduction of this article is prohibited. 
For $n=2$, since $u_{1}^{\alpha, \beta}$ and $u_{2}^{\alpha, \beta}$ are radially symmetric, there exists $C_{3}>0$, independent of $\beta>0$, that for $\left.i=1,2,\left|u_{i}^{\alpha, \beta}\right|_{\infty,\{x \in \Omega \mid}|x| \geq R\right\} \leq C_{3}\left\|u_{i}^{\alpha, \beta}\right\|_{\lambda_{i},\{x \in \Omega|| x \mid \geq R\}}$. Then, by the same argument with the case $n=1$, there exists a constant $C_{4}>0$ such that for large $\alpha \# \beta^{\frac{3}{4}}$,

$$
\left|u_{i}^{\alpha, \beta}\right|_{\infty,\{x \in \Omega|| x \mid \geq R\}} \leq C_{4}\left(\frac{\beta^{\frac{3}{4}}}{\alpha}\right)^{\frac{1}{2}}=C_{4} \frac{\beta^{\frac{3}{8}}}{\sqrt{\alpha}}, \quad i=1,2 .
$$

For $n=3$, it suffices to prove that $\left|v_{1, c}^{\alpha, \beta}\right|_{\infty, \Omega}$ and $\left|v_{2, c}^{\alpha, \beta}\right|_{\infty, \Omega}$ is bounded for large $\alpha \# \beta^{\frac{1}{2}}$. Since $\left(v_{1, c}^{\alpha, \beta}, v_{2, c}^{\alpha, \beta}\right)$ satisfies

$$
\begin{gathered}
\Delta v_{1, c}^{\alpha, \beta}-\frac{\lambda_{1}}{\beta} v_{1, c}^{\alpha, \beta}+\frac{\beta_{11}}{\alpha}\left(v_{1, c}^{\alpha, \beta}\right)^{3}+\beta_{12} v_{1, c}^{\alpha, \beta}\left(v_{2, c}^{\alpha, \beta}\right)^{2} \\
-\beta_{13} v_{1, c}^{\alpha, \beta}\left(u_{3}^{\alpha, \beta}\left(\beta^{-\frac{1}{2}} y\right)^{2}=0 \text { in } B_{\sqrt{\beta} R_{0}}(0)\right. \\
\Delta v_{2, c}^{\alpha, \beta}-\frac{\lambda_{2}}{\beta} v_{2, c}^{\alpha, \beta}+\beta_{21} v_{2, c}^{\alpha, \beta}\left(v_{1, c}^{\alpha, \beta}\right)^{2}+\frac{\beta_{22}}{\alpha}\left(v_{2, c}^{\alpha, \beta}\right)^{3} \\
-\beta_{23} v_{2, c}^{\alpha, \beta}\left(u_{3}^{\alpha, \beta}\left(\beta^{-\frac{1}{2}} y\right)\right)^{2}=0 \text { in } B_{\sqrt{\beta} R_{0}}(0), \\
-\Delta v_{1, c}^{\alpha, \beta} \leq \\
-\Delta v_{2, c}^{\alpha, \beta} \leq\left(\beta_{11}^{\alpha}\left(v_{1, c}^{\alpha, \beta}\right)^{2}+\beta_{12}^{\alpha, \beta}\left(v_{2, c}^{\alpha, \beta}\right)^{2}+\frac{\beta_{22}}{\alpha}\left(v_{2, c}^{\alpha, \beta}\right)^{2}\right) v_{1, c}^{\alpha, \beta} \quad \text { in } B_{\sqrt{\beta} R_{0}}^{\alpha, \beta}(0),
\end{gathered}
$$

Then, for each $l \geq 0$, we multiply both sides of (3.34) by $\left(v_{1, c}^{\alpha, \beta}\right)^{2 l+1}$, (3.35) by $\left(v_{2, c}^{\alpha, \beta}\right)^{2 l+1}$ and integrate parts and add two inequalities. Then for some $C>0$, independent of $\alpha, \beta>1, l \geq 0$,

$$
\begin{aligned}
& \int_{B_{\sqrt{\beta} R_{0}}(0)}\left|\nabla\left(v_{1, c}^{\alpha, \beta}\right)^{l+1}\right|^{2}+\left|\nabla\left(v_{2, c}^{\alpha, \beta}\right)^{l+1}\right|^{2} d y \\
& \leq C(l+1) \int_{B_{\sqrt{\beta} R_{0}}(0)}\left(v_{1, c}^{\alpha, \beta}\right)^{2 l+4}+\left(v_{1, c}^{\alpha, \beta}\right)^{2 l+2}\left(v_{2, c}^{\alpha, \beta}\right)^{2}+\left(v_{1, c}^{\alpha, \beta}\right)^{2}\left(v_{2, c}^{\alpha, \beta}\right)^{2 l+2}+\left(v_{2, c}^{\alpha, \beta}\right)^{2 l+4} d y .
\end{aligned}
$$

Then, using the Sobolev inequality and Hölder's inequality, we see that for some $C^{\prime}>0$, independent of $l \geq 0$,

$$
\begin{aligned}
\left(\int_{B_{\sqrt{\beta} R_{0}}(0)}\left(v_{1, c}^{\alpha, \beta}\right)^{6(l+1)}+\left(v_{2, c}^{\alpha, \beta}\right)^{6(l+1)} d y\right)^{\frac{1}{3}} & \leq C^{\prime}(l+1) \int_{B_{\sqrt{\beta} R_{0}}(0)}\left(v_{1, c}^{\alpha, \beta}\right)^{2 l+4} \\
& +\left(v_{2, c}^{\alpha, \beta}\right)^{2 l+4} d y .
\end{aligned}
$$

In view of (3.32), we get that $\sum_{i=1}^{2}\left|\nabla v_{i, c}^{\alpha, \beta}\right|_{2, \Omega_{c}^{\beta}}^{2}$ is bounded for large $\alpha \# \beta^{\frac{1}{2}}$. Then we see that for any $q \geq 6, \int_{B_{\sqrt{\beta} R_{0}}(0)}\left(v_{1, c}^{\alpha, \beta}\right)^{q}+\left(v_{2, c}^{\alpha, \beta}\right)^{q} d y$ is bounded for large $\alpha \# \beta^{\frac{1}{2}}$. Then, applying Theorems 9.20 and 9.26 in [13], we get the boundedness of $\left|v_{1, c}^{\alpha, \beta}\right|_{\infty, B \sqrt{\beta} R_{0}}(0)$ and $\left|v_{2, c}^{\alpha, \beta}\right|_{\infty, B \sqrt{\beta} R_{0}}(0)$ for large $\alpha \# \beta^{\frac{1}{2}}$. These complete the proof of Proposition 6.

Copyright $@$ by SIAM. Unauthorized reproduction of this article is prohibited. 
3.3. Finer estimates for the renormalized equations. From now on, we assume that $\alpha \# \beta^{1+\delta_{n}} \rightarrow \infty$ for $\delta_{1}, \delta_{2} \geq \frac{1}{4}$ if $n=1,2$, and $\delta_{3}>0$ if $n=3$. We note that

$$
\alpha \# \beta^{1+\delta_{n}} \rightarrow \infty \Longrightarrow\left\{\begin{array}{l}
\alpha \# \beta^{\frac{3}{4}} \rightarrow \infty \text { if } n=1,2, \\
\alpha \# \beta^{\frac{1}{2}} \rightarrow \infty \text { if } n=3 .
\end{array}\right.
$$

Now, from Proposition 3, we see that for the least energy solution $U_{3}$ of (1.4), $u_{3}^{\alpha, \beta} \rightarrow$ $U_{3}$ in $H_{0}^{1}(\Omega)$ as $\alpha \# \beta^{1+\delta_{n}} \rightarrow \infty$. In order to prove Theorem 1 , it is required to get $u_{3}^{\alpha, \beta} \rightarrow U_{3}$ in $C^{1}$ near the boundary for $n=1,2$, but $u_{3}^{\alpha, \beta} \rightarrow U_{3}$ in $C^{0}$ near the center for $n=3$.

Proposition 7. We take any $R \in\left(0, R_{0}\right)$. For any $\delta_{1} \geq \frac{1}{4}, \delta_{2} \geq \frac{1}{4}$, and $\delta_{3}>0$,

(i) $u_{3}^{\alpha, \beta} \rightarrow U_{3}$ in $C^{1}(\bar{\Omega})$ as $\alpha \# \beta^{1+\delta_{n}} \rightarrow \infty$ if $n=1$;

(ii) $u_{3}^{\alpha, \beta} \rightarrow U_{3}$ in $C^{1}(\overline{\{x \in \Omega|| x \mid \geq R\}})$ as $\alpha \# \beta^{1+\delta_{n}} \rightarrow \infty$ if $n=2$;

(iii) $u_{3}^{\alpha, \beta} \rightarrow U_{3}$ in $C(\bar{\Omega})$ as $\alpha \# \beta^{1+\delta_{n}} \rightarrow \infty$ if $n=3$.

Proof. Note that $u_{3}^{\alpha, \beta}$ and $U_{3}$ satisfy

$$
\begin{aligned}
\Delta u_{3}^{\alpha, \beta}-\lambda_{3} u_{3}^{\alpha, \beta}+\beta_{33}\left(u_{3}^{\alpha, \beta}\right)^{3}=\left(\beta \beta_{13}\left(u_{1}^{\alpha, \beta}\right)^{2}+\beta \beta_{23}\left(u_{2}^{\alpha, \beta}\right)^{2}\right) u_{3}^{\alpha, \beta} \text { in } \Omega, \\
\Delta U_{3}-\lambda_{3} U_{3}+\beta_{33}\left(U_{3}\right)^{3}=0 \quad \text { in } \quad \Omega .
\end{aligned}
$$

Then, defining $w^{\alpha, \beta} \equiv u_{3}^{\alpha, \beta}-U_{3}$, we see

$$
-\Delta w^{\alpha, \beta}+\lambda_{3} w^{\alpha, \beta}=\beta_{33}\left(u_{3}^{\alpha, \beta}\right)^{3}-\beta_{33}\left(U_{3}\right)^{3}-\left(\beta \beta_{13}\left(u_{1}^{\alpha, \beta}\right)^{2}+\beta \beta_{23}\left(u_{2}^{\alpha, \beta}\right)^{2}\right) u_{3}^{\alpha, \beta} .
$$

We define $f \equiv \beta_{33}\left(u_{3}^{\alpha, \beta}\right)^{3}-\beta_{33}\left(U_{3}\right)^{3}, \quad g \equiv\left(\beta \beta_{13}\left(u_{1}^{\alpha, \beta}\right)^{2}+\beta \beta_{23}\left(u_{2}^{\alpha, \beta}\right)^{2}\right) u_{3}^{\alpha, \beta}$. By Proposition $3,\left\{\left|u_{3}^{\alpha, \beta}\right|_{\infty, \Omega}\right\}_{\alpha \geq 1, \beta>0}$ is bounded. Thus, for any $p>1$, there exists a constant $C>0$, independent of large $\alpha, \beta>0$ such that

$$
|f|_{p, \Omega} \leq C\left|w^{\alpha, \beta}\right|_{p, \Omega}, \quad|g|_{p, \Omega} \leq C \beta\left|\left(u_{1}^{\alpha, \beta}\right)^{2} u_{3}^{\alpha, \beta}\right|_{p, \Omega}+C \beta\left|\left(u_{2}^{\alpha, \beta}\right)^{2} u_{3}^{\alpha, \beta}\right|_{p, \Omega} .
$$

For $n=1$, we take $p=2$. Then, we see from (3.33) and Proposition 6 that for some $C^{\prime}>0$, independent of large $\alpha, \beta>0$,

$$
|g|_{2, \Omega} \leq C \beta \sum_{i=1}^{2}\left|u_{i}^{\alpha, \beta}\right|_{\infty, \Omega}\left|u_{i}^{\alpha, \beta} u_{3}^{\alpha, \beta}\right|_{2, \Omega} \leq C^{\prime} \beta \frac{\beta^{\frac{3}{8}}}{\alpha^{\frac{1}{2}}} \frac{1}{\alpha^{\frac{1}{2}} \beta^{\frac{1}{8}}}=C^{\prime} \frac{\beta^{1+\frac{1}{4}}}{\alpha} .
$$

Together with Proposition 3 , we see that $|f|_{2, \Omega}$ and $|g|_{2, \Omega}$ converge to 0 as $\alpha \# \beta^{1+\frac{1}{4}} \rightarrow$ $\infty$. Then it follows from the $W^{2,2}$-estimate $\left[13\right.$, Theorem 8.12] that $w^{\alpha, \beta} \rightarrow 0$ in $W^{2,2}(\Omega)$ as $\alpha \# \beta^{1+\frac{1}{4}} \rightarrow \infty$. This implies that $w^{\alpha, \beta} \rightarrow 0$ in $C^{1}(\bar{\Omega})$ as $\alpha \# \beta^{1+\frac{1}{4}} \rightarrow \infty$.

For $n=2$, we take $p=2$. Then, we see from Proposition 3, (3.33), and Proposition 6 that for some $C^{\prime}>0$, independent of large $\alpha, \beta>0$,

$$
\left.|g|_{2,\{x \in \Omega|| x \mid \geq R\}} \leq C \beta \sum_{i=1}^{2}\left|u_{i}^{\alpha, \beta}\right|_{\infty,\{x \in \Omega}|| x \mid \geq R\right\}\left|u_{i}^{\alpha, \beta} u_{3}^{\alpha, \beta}\right|_{2, \Omega} \leq C^{\prime} \frac{\beta^{1+\frac{1}{4}}}{\alpha} .
$$

By the same arguments for $n=1$, we see that $w^{\alpha, \beta} \rightarrow 0$ in $W^{2,2}(\{x \in \Omega|| x \mid \geq 2 R\})$ as $\alpha \# \beta^{1+\frac{1}{4}} \rightarrow \infty$. Since $w^{\alpha, \beta}$ is radially symmetric, we get that

$$
w^{\alpha, \beta} \rightarrow 0 \text { in } C^{1}(\overline{\{x \in \Omega|| x \mid \geq 2 R\}}) \text { as } \alpha \# \beta^{1+\frac{1}{4}} \rightarrow \infty .
$$

Copyright $@$ by SIAM. Unauthorized reproduction of this article is prohibited. 
For $n=3$, we take any $p>\frac{3}{2}$. Then, we see from Propositions 3,5 , and 6 that for some $C^{\prime}>0$, independent of large $\alpha, \beta>0$,

$$
|g|_{p, \Omega} \leq C \beta \sum_{i=1}^{2}\left(\left|u_{i}^{\alpha, \beta}\right|_{\infty, \Omega}\right)^{\frac{2 p-2}{p}}\left(\left|u_{i}^{\alpha, \beta} u_{3}^{\alpha, \beta}\right|_{2, \Omega}\right)^{\frac{2}{p}} \leq C^{\prime} \beta \frac{\beta^{\frac{2 p-2}{2 p}}}{\alpha^{\frac{2 p-2}{2 p}}} \frac{1}{\alpha^{\frac{1}{p}} \beta^{\frac{1}{2 p}}}=C^{\prime} \frac{\beta^{1+\frac{2 p-3}{2 p}}}{\alpha} .
$$

Thus, it follows from Lemma 9.17 and Theorem 9.19 in [13] that

$$
w^{\alpha, \beta} \rightarrow 0 \quad \text { in } \quad W^{2, p}(\Omega) \quad \text { as } \quad \alpha \# \beta^{1+\frac{2 p-3}{2 p}} \rightarrow \infty .
$$

Since $W^{2, p}(\Omega) \hookrightarrow C(\bar{\Omega})$ for $p>\frac{3}{2}$, it follows that $w^{\alpha, \beta} \rightarrow 0$ in $C(\bar{\Omega})$ as $\alpha \# \beta^{1+\frac{2 p-3}{2 p}} \rightarrow$ $\infty$. Since $1+\frac{2 p-3}{2 p}=1$ for $p=\frac{3}{2}$, we conclude that for any $\delta_{3}>0, w^{\alpha, \beta} \rightarrow 0$ in $C(\bar{\Omega})$ as $\alpha \# \beta^{1+\delta_{3}} \rightarrow \infty$. This completes the proof.

Proposition 8. (i) There is a constant $C>0$ such that for large $\alpha \# \beta^{1+\delta_{n}}$,

$$
\left\{\begin{array}{l}
\sum_{i=1}^{2}\left(\left|\nabla v_{i, b}^{\alpha, \beta}\right|_{2, \Omega_{b}^{\beta}}+\left|v_{i, b}^{\alpha, \beta}\right|_{2, \Omega_{b}^{\beta}}\right) \leq C \text { if } n=1,2, \\
\sum_{i=1}^{2}\left(\left|\nabla v_{i, c}^{\alpha, \beta}\right|_{2, \Omega_{c}^{\beta}}+\left|v_{i, c}^{\alpha, \beta}\right|_{2, \Omega_{c}^{\beta}}\right) \leq C \text { if } n=3 .
\end{array}\right.
$$

(ii) There exists a constant $\delta>0$ such that

$$
\left\{\begin{array}{l}
\sum_{i=1}^{2}\left|v_{i, b}^{\alpha, \beta}\right|_{4, \Omega_{b}^{\beta}} \geq \delta \quad \text { and } \\
\lim _{\alpha \# \beta^{1+\delta_{n}} \rightarrow \infty} \beta^{\frac{5}{8}-\frac{n}{4}}\left(\sum_{i=1}^{2}\left|v_{i, c}^{\alpha, \beta}\right|_{4, \Omega_{c}^{\beta}}\right)^{2}=0 \quad \text { if } n=1,2, \\
\sum_{i=1}^{2}\left|v_{i, c}^{\alpha, \beta}\right|_{4, \Omega_{c}^{\beta}} \geq \delta \quad \text { and } \\
\lim _{\alpha \# \beta^{1+\delta_{n}} \rightarrow \infty} \beta^{-\frac{5}{8}+\frac{n}{4}}\left(\sum_{i=1}^{2}\left|v_{i, b}^{\alpha, \beta}\right|_{4, \Omega_{b}^{\beta}}\right)^{2}=0 \quad \text { if } n=3 .
\end{array}\right.
$$

Proof. (i) Let $\Omega_{B, R_{0}}=\left\{x \in \Omega \mid \operatorname{dist}(x, \partial \Omega) \leq \frac{R_{0}}{2}\right\}=\left\{x\left|\frac{R_{0}}{2} \leq\right| x \mid \leq R_{0}\right\}$. Since we have the convergence in Proposition 7, as in the proof of [7, Proposition 17], we deduce that for some $C>0$, independent of large $\alpha \# \beta^{1+\delta_{n}}$,

$$
\left|u_{i}^{\alpha, \beta}\right|_{2, \Omega_{B, R_{0}}}^{2} \leq C\left(\beta^{-\frac{1}{2}}\left|\nabla u_{i}^{\alpha, \beta}\right|_{2, \Omega_{B, R_{0}}}^{2}+\sqrt{\beta}\left|u_{3}^{\alpha, \beta} u_{i}^{\alpha, \beta}\right|_{2, \Omega_{B, R_{0}}}^{2}\right) \quad(i=1,2) .
$$

Then we have

$$
\sum_{i=1}^{2}\left|v_{i, b}^{\alpha, \beta}\right|_{2, \Omega_{b}^{\beta}}^{2} \leq C \sum_{i=1}^{2}\left[\left|\nabla v_{i, b}^{\alpha, \beta}\right|_{2, \Omega_{b}^{\beta}}^{2}+\sqrt{\beta} \int_{\Omega_{b}^{\beta}}\left(v_{3}^{\alpha, \beta}\left(R_{0}-\beta^{-\frac{1}{4}}|y|\right)\right)^{2}\left(v_{i, b}^{\alpha, \beta}\right)^{2} d y\right] .
$$

If $n=1,2$, then Proposition 5 implies the first claim of (i).

If $n=3$, then Proposition 5 implies that there is a constant $c>0$ satisfying

$$
\sum_{i=1}^{2}\left(\left|\nabla v_{i, c}^{\alpha, \beta}\right|_{2, \Omega_{c}^{\beta}}^{2}+\int_{\Omega_{c}^{\beta}}\left(v_{3}^{\alpha, \beta}\left(\beta^{-\frac{1}{2}} y\right)\right)^{2}\left(v_{i, c}^{\alpha, \beta}\right)^{2} d y\right) \leq c .
$$

In view of Proposition 7 and $\inf _{B_{\frac{R_{0}}{2}}(0)} U_{3}>0$, we get that $\inf _{\Omega_{c}^{\beta}} v_{3}^{\alpha, \beta}\left(\beta^{-\frac{1}{2}} y\right) \geq C$ for some constant $C>0$, and thus

$$
\begin{aligned}
& \sum_{i=1}^{2}\left(\left|\nabla v_{i, c}^{\alpha, \beta}\right|_{2, \Omega_{c}^{\beta}}^{2}+C^{2}\left|v_{i, c}^{\alpha, \beta}\right|_{2, \Omega_{c}^{\beta}}^{2}\right) \\
& \leq \sum_{i=1}^{2}\left(\left|\nabla v_{i, c}^{\alpha, \beta}\right|_{2, \Omega_{c}^{\beta}}^{2}+\int_{\Omega_{c}^{\beta}}\left(v_{3}^{\alpha, \beta}\left(\beta^{-\frac{1}{2}} y\right)\right)^{2}\left(v_{i, c}^{\alpha, \beta}\right)^{2} d y\right) \leq c .
\end{aligned}
$$

Copyright $@$ by SIAM. Unauthorized reproduction of this article is prohibited. 
Now we complete the proof of Proposition 8(i).

(ii) In view of (3.32), (3.37), and (3.39), there is a constant $c>1$ satisfying

$$
\begin{aligned}
& c \min \left\{\frac{\beta^{2-\frac{n}{2}}}{\alpha} \sum_{i=1}^{2}\left|v_{i, c}^{\alpha, \beta}\right|_{4, \Omega_{c}^{\beta}}^{4}+\frac{\beta^{\frac{3}{4}}}{\alpha} \sum_{i=1}^{2}\left|v_{i, b}^{\alpha, \beta}\right|_{4, \Omega_{b}^{\beta}}^{4}, \frac{\beta^{\frac{3}{4}}}{\alpha}, \frac{\beta^{2-\frac{n}{2}}}{\alpha}\right\} \\
& \geq \frac{\beta^{2-\frac{n}{2}}}{\alpha} \sum_{i=1}^{2}\left(\left|\nabla v_{i, c}^{\alpha, \beta}\right|_{2, \Omega_{c}^{\beta}}^{2}+\int_{\Omega_{c}^{\beta}}\left(v_{3}^{\alpha, \beta}\left(\beta^{-\frac{1}{2}} y\right)\right)^{2}\left(v_{i, c}^{\alpha, \beta}\right)^{2} d y\right) \\
& +\frac{\beta^{\frac{3}{4}}}{\alpha} \sum_{i=1}^{2}\left(\left|\nabla v_{i, b}^{\alpha, \beta}\right|_{2, \Omega_{b}^{\beta}}^{2}+\int_{\Omega_{b}^{\beta}} \sqrt{\beta}\left(v_{3}^{\alpha, \beta}\left(R_{0}-\beta^{-\frac{1}{4}}|y|\right)\right)^{2}\left(v_{i, b}^{\alpha, \beta}\right)^{2} d y\right) \\
& \geq \frac{1}{c}\left\{\frac{\beta^{2-\frac{n}{2}}}{\alpha} \sum_{i=1}^{2}\left(\left|\nabla v_{i, c}^{\alpha, \beta}\right|_{2, \Omega_{c}^{\beta}}^{2}+\left|v_{i, c}^{\alpha, \beta}\right|_{2, \Omega_{c}^{\beta}}^{2}\right)\right. \\
& \left.+\frac{\beta^{\frac{3}{4}}}{\alpha} \sum_{i=1}^{2}\left(\left|\nabla v_{i, b}^{\alpha, \beta}\right|_{2, \Omega_{b}^{\beta}}^{2}+\left|v_{i, b}^{\alpha, \beta}\right|_{2, \Omega_{b}^{\beta}}^{2}\right)\right\} .
\end{aligned}
$$

Together with the Sobolev inequality, we get that for some constant $C>0$,

$$
\begin{aligned}
& \min \left\{\left[\frac{\beta^{1-\frac{n}{4}}}{\alpha^{\frac{1}{2}}}\left(\sum_{i=1}^{2}\left|v_{i, c}^{\alpha, \beta}\right|_{4, \Omega_{c}^{\beta}}\right)^{2}+\frac{\beta^{\frac{1}{2}-\frac{1}{8}}}{\alpha^{\frac{1}{2}}}\left(\sum_{i=1}^{2}\left|v_{i, b}^{\alpha, \beta}\right|_{4, \Omega_{b}^{\beta}}\right)^{2}\right]^{2}, \frac{\beta^{\frac{3}{4}}}{\alpha}, \frac{\beta^{2-\frac{n}{2}}}{\alpha}\right\} \\
& \geq C\left\{\frac{\beta^{2-\frac{n}{2}}}{\alpha}\left(\sum_{i=1}^{2}\left|v_{i, c}^{\alpha, \beta}\right|_{4, \Omega_{c}^{\beta}}\right)^{2}+\frac{\beta^{\frac{3}{4}}}{\alpha}\left(\sum_{i=1}^{2}\left|v_{i, b}^{\alpha, \beta}\right|_{4, \Omega_{b}^{\beta}}\right)^{2}\right\} .
\end{aligned}
$$

If $n=1,2$, then $\frac{5}{4}-\frac{n}{2}>0$. Dividing (3.41) by $\frac{\beta^{\frac{3}{4}}}{\alpha}$, we get that

$$
\begin{aligned}
& \min \left\{\left[\beta^{\frac{5}{8}-\frac{n}{4}}\left(\sum_{i=1}^{2}\left|v_{i, c}^{\alpha, \beta}\right|_{4, \Omega_{c}^{\beta}}\right)^{2}+\left(\sum_{i=1}^{2}\left|v_{i, b}^{\alpha, \beta}\right|_{4, \Omega_{b}^{\beta}}\right)^{2}\right]^{2}, 1\right\} \\
& \geq C\left\{\beta^{\frac{5}{4}-\frac{n}{2}}\left(\sum_{i=1}^{2}\left|v_{i, c}^{\alpha, \beta}\right|_{4, \Omega_{c}^{\beta}}\right)^{2}+\left(\sum_{i=1}^{2}\left|v_{i, b}^{\alpha, \beta}\right|_{4, \Omega_{b}^{\beta}}\right)^{2}\right\} \\
& \geq C\left\{\beta^{\frac{5}{8}-\frac{n}{4}}\left(\sum_{i=1}^{2}\left|v_{i, c}^{\alpha, \beta}\right|_{4, \Omega_{c}^{\beta}}\right)^{2}+\left(\sum_{i=1}^{2}\left|v_{i, b}^{\alpha, \beta}\right|_{4, \Omega_{b}^{\beta}}\right)^{2}\right\} .
\end{aligned}
$$

Then we have

$$
\frac{1}{C} \geq \beta^{\frac{5}{8}-\frac{n}{4}}\left(\sum_{i=1}^{2}\left|v_{i, c}^{\alpha, \beta}\right|_{4, \Omega_{c}^{\beta}}\right)^{2}+\left(\sum_{i=1}^{2}\left|v_{i, b}^{\alpha, \beta}\right|_{4, \Omega_{b}^{\beta}}\right)^{2} \geq C,
$$

and thus

$$
\lim _{\alpha \# \beta^{1+\delta_{n}} \rightarrow \infty}\left(\sum_{i=1}^{2}\left|v_{i, c}^{\alpha, \beta}\right|_{4, \Omega_{c}^{\beta}}\right)^{2}=0 .
$$

Copyright (c) by SIAM. Unauthorized reproduction of this article is prohibited. 
The first and second line of (3.42) also implies that

$$
\begin{aligned}
& 2\left(\beta^{\frac{5}{4}-\frac{n}{2}}\left(\sum_{i=1}^{2}\left|v_{i, c}^{\alpha, \beta}\right|_{4, \Omega_{c}^{\beta}}\right)^{4}+\left(\sum_{i=1}^{2}\left|v_{i, b}^{\alpha, \beta}\right|_{4, \Omega_{b}^{\beta}}\right)^{4}\right) \\
& \geq C\left(\beta^{\frac{5}{4}-\frac{n}{2}}\left(\sum_{i=1}^{2}\left|v_{i, c}^{\alpha, \beta}\right|_{4, \Omega_{c}^{\beta}}\right)^{2}+\left(\sum_{i=1}^{2}\left|v_{i, b}^{\alpha, \beta}\right|_{4, \Omega_{b}^{\beta}}\right)^{2}\right) .
\end{aligned}
$$

Together with (3.43), we see that $\beta^{\frac{5}{4}-\frac{n}{2}}\left(\sum_{i=1}^{2}\left|v_{i, c}^{\alpha, \beta}\right|_{4, \Omega_{c}^{\beta}}\right)^{2}=O(1)$. By (3.44), we have

$$
\lim _{\alpha \# \beta^{1+\delta_{n} \rightarrow \infty}} \beta^{\frac{5}{8}-\frac{n}{4}}\left(\sum_{i=1}^{2}\left|v_{i, c}^{\alpha, \beta}\right|_{4, \Omega_{c}^{\beta}}\right)^{2}=\lim _{\alpha \# \beta^{1+\delta_{n} \rightarrow \infty}} O\left(\sum_{i=1}^{2}\left|v_{i, c}^{\alpha, \beta}\right|_{4, \Omega_{c}^{\beta}}\right)=0 .
$$

In view of (3.43), we get that $\lim _{\alpha \# \beta^{1+\delta_{n}} \rightarrow \infty}\left(\sum_{i=1}^{2}\left|v_{i, b}^{\alpha, \beta}\right|_{4, \Omega_{b}^{\beta}}\right)^{2} \geq C$ if $n=1,2$. The similar arguments also hold for the case $n=3$. Now we complete the proof of Proposition 8(ii).

Proposition 9. There is a constant $C \geq 1$ satisfying for large $\alpha \# \beta^{1+\delta_{n}}$,

$$
\begin{cases}\frac{1}{C} \leq\left\|v_{i, b}^{\alpha, \beta}\right\|_{L^{\infty}\left(\Omega_{b}^{\beta}\right)} \leq C & i=1,2 \quad \text { if } \quad n=1,2 \\ \frac{1}{C} \leq\left\|v_{i, c}^{\alpha, \beta}\right\|_{L^{\infty}\left(\Omega_{c}^{\beta}\right)} \leq C & i=1,2 \quad \text { if } \quad n=3\end{cases}
$$

Moreover,

$$
\left\{\begin{array}{l}
\lim _{\alpha \# \beta^{1+\delta_{n}} \rightarrow \infty}\left(\sum_{i=1}^{2}\left\|v_{i, c}^{\alpha, \beta}\right\|_{L^{\infty}\left(\Omega_{c}^{\beta}\right)}\right)=\lim _{\alpha \# \beta^{1+\delta_{n}} \rightarrow \infty} O\left(\beta^{\frac{n}{4}-\frac{5}{8}}\right)=0 \text { if } n=1,2, \\
\lim _{\alpha \# \beta^{1+\delta_{n}} \rightarrow \infty}\left(\sum_{i=1}^{2}\left\|v_{i, b}^{\alpha, \beta}\right\|_{L^{\infty}\left(\Omega_{b}^{\beta}\right)}\right)=\lim _{\alpha \# \beta^{1+\delta_{n}} \rightarrow \infty} O\left(\beta^{-\frac{n}{4}+\frac{5}{8}}\right)=0 \text { if } n=3 .
\end{array}\right.
$$

Proof. First, we consider the case $n=3$. In view of Propositions 5 and 8 and (3.40), we have for some $c \geq 1$,

$$
\begin{aligned}
c & \geq \frac{\left|v_{1, c}^{\alpha, \beta}\right|_{4, \Omega_{c}^{\beta}}^{4}}{\alpha}+\frac{\left|v_{2, c}^{\alpha, \beta}\right|_{4, \Omega_{c}^{\beta}}^{4}}{\alpha}+\left|v_{1, c}^{\alpha, \beta} v_{2, c}^{\alpha, \beta}\right|_{2, \Omega_{c}^{\beta}}^{2}+\beta^{-\frac{5}{4}+\frac{n}{2}} \sum_{i=1}^{2}\left|v_{i, b}^{\alpha, \beta}\right|_{4, \Omega_{b}^{\beta}}^{4} \\
& \geq \frac{1}{c} \sum_{i=1}^{2}\left\{\left(\left|\nabla v_{i, c}^{\alpha, \beta}\right|_{2, \Omega_{c}^{\beta}}^{2}+\left|v_{i, c}^{\alpha, \beta}\right|_{2, \Omega_{c}^{\beta}}^{2}\right)+\beta^{-\frac{5}{4}+\frac{n}{2}}\left(\left|\nabla v_{i, b}^{\alpha, \beta}\right|_{2, \Omega_{b}^{\beta}}^{2}+\left|v_{i, b}^{\alpha, \beta}\right|_{2, \Omega_{b}^{\beta}}^{2}\right)\right\} .
\end{aligned}
$$

We recall from Proposition 8 that

$$
\lim _{\alpha \# \beta^{1+\delta_{n}} \rightarrow \infty} \beta^{-\frac{5}{8}+\frac{n}{4}}\left(\sum_{i=1}^{2}\left|v_{i, b}^{\alpha, \beta}\right|_{4, \Omega_{b}^{\beta}}\right)^{2}=0 .
$$

Suppose that $\liminf \operatorname{in}_{\alpha \beta^{1+\delta_{n} \rightarrow \infty}}\left|v_{1, c}^{\alpha, \beta}\right|_{\infty, \Omega_{c}^{\beta}}=0$ or $\liminf _{\alpha \# \beta^{1+\delta_{n} \rightarrow \infty}}\left|v_{2, c}^{\alpha, \beta}\right|_{\infty, \Omega_{c}^{\beta}}=0$, we see from (3.47) and (3.48) that

$$
\liminf _{\alpha \# \beta^{1+\delta_{n}} \rightarrow \infty} \sum_{i=1}^{2}\left(\left|\nabla v_{i, c}^{\alpha, \beta}\right|_{2, \Omega_{c}^{\beta}}^{2}+\left|v_{i, c}^{\alpha, \beta}\right|_{2, \Omega_{c}^{\beta}}^{2}\right)=0
$$

which implies that $\lim _{\alpha \# \beta^{1+\delta_{n} \rightarrow \infty}}\left(\sum_{i=1}^{2}\left|v_{i, c}^{\alpha, \beta}\right|_{4, \Omega_{c}^{\beta}}^{4}\right)=0$ by the Sobolev inequality. This contradicts Proposition 8 , and thus there is a constant $C>0$ such that for large

Copyright (c) by SIAM. Unauthorized reproduction of this article is prohibited. 
$\alpha \# \beta^{1+\delta_{n}},\left\|v_{i, c}^{\alpha, \beta}\right\|_{L^{\infty}\left(\Omega_{c}^{\beta}\right)} \geq C, i=1,2$. We also note that Proposition 6 implies that $v_{i, c}^{\alpha, \beta}$ is uniformly bounded from above in $L^{\infty}\left(\Omega_{b}^{\beta}\right)$. Therefore, the proof of (3.45) for $n=3$ is obtained. Note that $-\frac{5}{4}+\frac{n}{2}>0$ if $n=3$. Then (3.47) and the Sobolev imbedding theorem imply the estimation (3.46) for $n=3$.

Second, we consider cases $n=1,2$. In view of Propositions 8 and 5 and (3.40), we have for some $c \geq 1$,

$$
\begin{aligned}
& c \geq \beta^{\frac{5}{4}-\frac{n}{2}} \sum_{i=1}^{2}\left|v_{i, c}^{\alpha, \beta}\right|_{4, \Omega_{c}^{\beta}}^{4}+\frac{\left|v_{1, b}^{\alpha, \beta}\right|_{4, \Omega_{b}^{\beta}}^{4}}{\alpha}+\frac{\left|v_{2, b}^{\alpha, \beta}\right|_{4, \Omega_{b}^{\beta}}^{4}}{\alpha}+\left|v_{1, b}^{\alpha, \beta} v_{2, b}^{\alpha, \beta}\right|_{2, \Omega_{b}^{\beta}}^{2} \\
& \geq \frac{1}{c} \sum_{i=1}^{2}\left\{\beta^{\frac{5}{4}-\frac{n}{2}}\left(\left|\nabla v_{i, c}^{\alpha, \beta}\right|_{2, \Omega_{c}^{\beta}}^{2}+\left|v_{i, c}^{\alpha, \beta}\right|_{2, \Omega_{c}^{\beta}}^{2}\right)+\left(\left|\nabla v_{i, b}^{\alpha, \beta}\right|_{2, \Omega_{b}^{\beta}}^{2}+\left|v_{i, b}^{\alpha, \beta}\right|_{2, \Omega_{b}^{\beta}}^{2}\right)\right\} .
\end{aligned}
$$

By a similar argument with the case $n=3$ above, there is a constant $C>0$ such that $\left\|v_{i, b}^{\alpha, \beta}\right\|_{L^{\infty}\left(\Omega_{b}^{\beta}\right)} \geq C$ for $i=1,2$. In view of (3.49) and the Sobolev imbedding theorem, we also note that $v_{i, b}^{\alpha, \beta}$ is uniformly bounded from above in $L^{\infty}\left(\Omega_{b}^{\beta}\right)$.

If $n=1$, then the Sobolev imbedding theorem and (3.49) imply the estimation (3.46). If $n=2$, then the Sobolev imbedding theorem and (3.49) imply $\lim _{\alpha \# \beta^{1+\delta_{n}} \rightarrow \infty} \mid$ $\left.v_{i, c}^{\alpha, \beta}\right|_{p, \Omega_{c}^{\beta}}=0$ for any $p>1$. By applying $W^{2, p}$ estimates to (3.31), we see that the estimation (3.46) holds. Now we complete the proof of Proposition 9.

Proposition 10. There exists a constant $R>0$ such that

$$
\left\{\begin{array}{l}
\liminf \operatorname{in}_{\alpha \beta^{1+\delta_{n}} \rightarrow \infty}\left(\sum_{i=1}^{2} \int_{0}^{R}\left|v_{i, b}^{\alpha, \beta}\right|^{4} d y\right)>0 \text { if } n=1,2, \\
\liminf { }_{\alpha \# \beta^{1+\delta_{n}} \rightarrow \infty}\left(\sum_{i=1}^{2} \int_{B_{R}(0)}\left|v_{i, c}^{\alpha, \beta}\right|^{4} d y\right)>0 \text { if } n=3 .
\end{array}\right.
$$

Proof. First, we consider cases $n=1,2$. Suppose that for any fixed $R>0$,

$$
\lim _{\alpha \# \beta^{1+\delta_{n}} \rightarrow \infty}\left(\sum_{i=1}^{2} \int_{0}^{R}\left|v_{i, b}^{\alpha, \beta}\right|^{4} d y\right)=0 .
$$

Since $v_{3}^{\alpha, \beta}\left(R_{0}\right)=0$ and $\left.\frac{d v_{3}^{\alpha, \beta}}{d r}\right|_{r=R_{0}}<0$, there exists a constant $c>0$ such that

$$
\sqrt{\beta}\left(v_{3}^{\alpha, \beta}\left(R_{0}-\beta^{-\frac{1}{4}}|y|\right)\right)^{2} \geq c|y|^{2} \quad \text { for } \quad 0 \leq|y| \leq \frac{\beta^{\frac{1}{4}} R_{0}}{2} .
$$

In view of Proposition 5, we have

$$
\int_{R \leq s \leq \frac{\beta^{\frac{1}{4}} R_{0}}{2}} \sum_{i=1}^{2}\left|v_{i, b}^{\alpha, \beta}\right|^{2} d s=O\left(R^{-2}\right) .
$$

By (3.51), (3.52), and Proposition 9, we get that $\lim _{\alpha \# \beta^{1+\delta_{n}} \rightarrow \infty}\left(\int_{\Omega_{b}^{\beta}} \sum_{i=1}^{2}\left|v_{i, b}^{\alpha, \beta}\right|^{4} d y\right)=$ 0 , which contradicts Proposition 8. This implies that there is a constant $R>0$ satisfying

$$
\lim _{\alpha \# \beta^{1+\delta_{n}} \rightarrow \infty}\left(\sum_{i=1}^{2} \int_{0}^{R}\left|v_{i, b}^{\alpha, \beta}\right|^{4} d y\right)>0 \quad \text { if } n=1,2 .
$$

Copyright (c) by SIAM. Unauthorized reproduction of this article is prohibited. 
Second, we consider a case $n=3$. From (3.31), we have

$$
\left\{\begin{aligned}
\Delta v_{1, c}^{\alpha, \beta} & =O(1)\left(\left|v_{1, c}^{\alpha, \beta}\right|+\left|v_{2, c}^{\alpha, \beta}\right|\right) \\
\Delta v_{2, c}^{\alpha, \beta} & =O(1)\left(\left|v_{1, c}^{\alpha, \beta}\right|+\left|v_{2, c}^{\alpha, \beta}\right|\right) .
\end{aligned}\right.
$$

To the contrary, suppose that for any fixed $R>0$,

$$
\lim _{\alpha \# \beta^{1+\delta_{n}} \rightarrow \infty}\left(\sum_{i=1}^{2} \int_{B_{R}(0)}\left|v_{i, c}^{\alpha, \beta}\right|^{4} d y\right)=\lim _{\alpha \# \beta^{1+\delta_{n}} \rightarrow \infty}\left(\sum_{i=1}^{2} \int_{0}^{R}\left|v_{i, c}^{\alpha, \beta}\right|^{4} s^{n-1} d s\left|S^{n-1}\right|\right)=0 .
$$

Then $W^{2, p}$-estimates and (3.53) imply that

$$
\lim _{\alpha \# \beta^{1+\delta_{n}} \rightarrow \infty}\left(\sum_{i=1}^{2}\left\|v_{i, c}^{\alpha, \beta}\right\|_{L^{\infty}\left(B_{\frac{R}{2}}(0)\right)}\right)=0 \text { for any fixed } R>0 .
$$

In view of Proposition 8, we see that $\int_{\frac{R}{2} \leq s \leq \frac{\sqrt{\beta} R_{0}}{2}} \sum_{i=1}^{2}\left(\left|\frac{d v_{i, c}^{\alpha, \beta}}{d s}\right|^{2}+\left|v_{i, c}^{\alpha, \beta}\right|^{2}\right) s^{n-1} d s \leq C$ for some constant $C>0$; thus

$$
\int_{\frac{R}{2} \leq s \leq \frac{\sqrt{\beta} R_{0}}{2}} \sum_{i=1}^{2}\left(\left|\frac{d v_{i, c}^{\alpha, \beta}}{d s}\right|^{2}+\left|v_{i, c}^{\alpha, \beta}\right|^{2}\right) d s=O\left(R^{-(n-1)}\right) .
$$

By Sobolev inequality and (3.54), we get that $\lim _{\alpha \# \beta^{1+\delta_{n}} \rightarrow \infty} \sum_{i=1}^{2}\left\|v_{i, c}^{\alpha, \beta}\right\|_{L^{\infty}\left(\Omega_{c}^{\beta}\right)}=0$, which contradicts Proposition 9. Thus there is a constant $R>0$ such that for $n=3$,

$$
\lim _{\alpha \# \beta^{1+\delta_{n}} \rightarrow \infty}\left(\sum_{i=1}^{2} \int_{B_{R}(0)}\left|v_{i, c}^{\alpha, \beta}\right|^{4} d y\right)>0 .
$$

We are going to improve the estimations (3.52) and (3.55).

Proposition 11. There are constants $c, C>0$, independent of large $\alpha \# \beta^{1+\delta_{n}}$, satisfying

$$
\begin{cases}v_{i, b}^{\alpha, \beta}(y) \leq C \exp (-c|y|) & \text { for } 0 \leq|y| \leq \beta^{\frac{1}{4}} R_{0} \quad \text { if } n=1,2 \\ v_{i, c}^{\alpha, \beta}(y) \leq C \exp (-c|y|) & \text { for } 0 \leq|y| \leq \sqrt{\beta} R_{0} \quad \text { if } \quad n=3\end{cases}
$$

Proof. We note from (3.29) that

$$
\frac{d^{2} v_{i, b}^{\alpha, \beta}}{d s^{2}}-\frac{(n-1) \beta^{-\frac{1}{4}}}{R_{0}-\beta^{-\frac{1}{4}} s} \frac{d v_{i, b}^{\alpha, \beta}}{d s} \geq-C\left(v_{1, b}^{\alpha, \beta}+v_{2, b}^{\alpha, \beta}\right) \text { in } \Omega_{b}^{\beta}, \quad i=1,2 .
$$

Together with (3.52) and [13, Theorem 9.20], we have

$$
\lim _{R \rightarrow+\infty} \sum_{i=1}^{2}\left\|v_{i, b}^{\alpha, \beta}\right\|_{L^{\infty}\left(R \leq|y| \leq \beta^{\left.\frac{1}{4} \frac{R_{0}}{2}\right)}\right.}=0 \text { if } n=1,2 .
$$

Moreover, (3.55) and the Sobolev inequality imply that

$$
\lim _{R \rightarrow+\infty} \sum_{i=1}^{2}\left\|v_{i, c}^{\alpha, \beta}\right\|_{L^{\infty}\left(R \leq|y| \leq \sqrt{\beta} \frac{R_{0}}{2}\right)}=0 \text { if } n=3 .
$$

Copyright (c) by SIAM. Unauthorized reproduction of this article is prohibited. 
In view of (3.28) and (3.30), we have

$$
v_{i, b}^{\alpha, \beta}\left(\beta^{\frac{1}{4}}\left(R_{0}-|x|\right)\right)=\beta^{\frac{1}{4}} v_{i, c}^{\alpha, \beta}(\sqrt{\beta} x) .
$$

By (3.46), we see that for $i=1,2$,

$$
\left\{\begin{array}{l}
\left\|v_{i, b}^{\alpha, \beta}\right\|_{L^{\infty}\left(\beta^{\frac{1}{4}} \frac{R_{0}}{2} \leq|y| \leq \beta^{\frac{1}{4}} R_{0}\right)}=\beta^{\frac{1}{4}}\left\|v_{i, c}^{\alpha, \beta}\right\|_{L^{\infty}\left(\Omega_{c}^{\beta}\right)}=O\left(\beta^{\frac{2 n-3}{8}}\right)=o(1) \text { if } n=1, \\
\left\|v_{i, b}^{\alpha, \beta}\right\|_{L^{\infty}\left(\beta^{\frac{1}{4}} \frac{R_{0}}{2} \leq|y| \leq \beta^{\frac{1}{4}} R_{0}\right)}=\beta^{\frac{1}{4}}\left\|v_{i, c}^{\alpha, \beta}\right\|_{L^{\infty}\left(\Omega_{c}^{\beta}\right)}=O\left(\beta^{\frac{2 n-3}{8}}\right)=O\left(\beta^{\frac{1}{8}}\right) \text { if } n=2, \\
\left\|v_{i, c}^{\alpha, \beta}\right\|_{L^{\infty}\left(\sqrt{\beta} \frac{R_{0}}{2} \leq|y| \leq \sqrt{\beta} R_{0}\right)}=\beta^{-\frac{1}{4}}\left\|v_{i, b}^{\alpha, \beta}\right\|_{L^{\infty}\left(\Omega_{b}^{\beta}\right)}=O\left(\beta^{\frac{3-2 n}{8}}\right)=o(1) \text { if } n=3 .
\end{array}\right.
$$

Now we shall prove (3.56).

First, we consider cases $n=1,2$. Although our proof is based on [7], we need more careful analysis in detail since $\left\|v_{i, b}^{\alpha, \beta}\right\|_{L^{\infty}\left(|y| \leq \beta^{\frac{1}{4}} R_{0}\right)}$ might not be sufficiently small for $n=2$ due to (3.61). Let $w_{i}^{\alpha, \beta}(x)=v_{i, b}^{\alpha, \beta}\left(\beta^{\frac{1}{4}} R_{0}-|x|\right)$ for $i=1,2$ and $w_{3}^{\alpha, \beta}(x)=$ $v_{3}^{\alpha, \beta}(x)$. For the exponential decay property of $w_{1}^{\alpha, \beta}$ and $w_{2}^{\alpha, \beta}$, we take the first eigenfunction $\varphi>0$ in $\Omega$ of $-\Delta$ with $\varphi=0$ on $\partial \Omega$ and $\max _{x \in \Omega} \varphi(x)=1$. Let $\mu_{1}>0$ be the corresponding first eigenvalue. For $a>0$, which will be determined later, we define

$$
\Phi(y) \equiv \exp \left(-a\left(\beta^{\frac{1}{4}} \varphi\left(\beta^{-\frac{1}{4}} y\right)\right)^{2}\right), \quad y=(\beta)^{\frac{1}{4}} x \in \Omega_{\beta}^{*}=\left\{y=(\beta)^{\frac{1}{4}} x \mid x \in \Omega\right\} .
$$

We note that

$$
-\Delta w_{1}^{\alpha, \beta}+\left(\frac{\lambda_{1}}{\sqrt{\beta}}+\beta^{\frac{1}{2}} \beta_{13}\left(w_{3}^{\alpha, \beta}\left(\beta^{-\frac{1}{4}} y\right)\right)^{2}-\frac{\beta_{11}}{\alpha}\left(w_{1}^{\alpha, \beta}\right)^{2}-\beta_{12}\left(w_{2}^{\alpha, \beta}\right)^{2}\right) w_{1}^{\alpha, \beta}=0 \text { in } \Omega_{\beta}^{*},
$$

and from Proposition 7 that for some $e_{1}>0$, independent of $j \geq 1$,

$$
\beta^{\frac{1}{2}} \beta_{13}\left(w_{3}^{\alpha, \beta}\left(\beta^{-\frac{1}{4}} y\right)\right)^{2} \geq e_{1}\left(d\left(y, \Omega_{\beta}^{*}\right)\right)^{2}, \quad y \in \Omega_{\beta}^{*} .
$$

We also note that

$$
-\Delta \Phi(y)=\left(-2 a \mu_{1}(\varphi(x))^{2}+2 a\left|\nabla_{x} \varphi(x)\right|^{2}-4 a^{2} \beta^{\frac{1}{2}}(\varphi(x))^{2}\left|\nabla_{x} \varphi(x)\right|^{2}\right) \Phi(y),
$$

and that for some $e_{2}>0$, independent of $\beta \geq 1$,

$$
\beta^{\frac{1}{2}}(\varphi(x))^{2}=\beta^{\frac{1}{2}}\left(\varphi\left(\beta^{-\frac{1}{4}} y\right)\right)^{2} \leq e_{2}\left(d\left(y, \Omega_{\beta}^{*}\right)\right)^{2}, \quad y \in \Omega_{\beta}^{*} .
$$

Then, for $e_{3} \equiv \max _{x \in \Omega}|\nabla \varphi(x)|^{2}$,

$$
\begin{aligned}
- & \Delta \Phi+\left(\frac{\lambda_{1}}{\sqrt{\beta}}+\beta^{\frac{1}{2}} \beta_{13}\left(w_{3}^{\alpha, \beta}\left(\beta^{-\frac{1}{4}} y\right)\right)^{2}-\frac{\beta_{11}}{\alpha}\left(w_{1}^{\alpha, \beta}\right)^{2}-\beta_{12}\left(w_{2}^{\alpha, \beta}\right)^{2}\right) \Phi \\
= & \left(\frac{\lambda_{1}}{\sqrt{\beta}}+\beta^{\frac{1}{2}} \beta_{13}\left(w_{3}^{\alpha, \beta}\left(\beta^{-\frac{1}{4}} y\right)\right)^{2}-\frac{\beta_{11}}{\alpha}\left(w_{1}^{\alpha, \beta}\right)^{2}-\beta_{12}\left(w_{2}^{\alpha, \beta}\right)^{2}\right) \Phi \\
& -\left(2 a \mu_{1}\left(\varphi\left(\beta^{-\frac{1}{4}} y\right)\right)^{2}-2 a\left|\nabla_{x} \varphi\left(\beta^{-\frac{1}{4}} y\right)\right|^{2}+4 a^{2}(\beta)^{\frac{1}{2}}\left(\varphi\left(\beta^{-\frac{1}{4}} y\right)\right)^{2}\left|\nabla_{x} \varphi\left(\beta^{-\frac{1}{4}} y\right)\right|^{2}\right) \Phi \\
\geq & \left(e_{1}\left(d\left(y, \Omega_{\beta}^{*}\right)\right)^{2}-4 a^{2} e_{2} e_{3}\left(d\left(y, \Omega_{\beta}^{*}\right)\right)^{2}\right) \Phi \\
& -\left(\frac{\beta_{11}}{\alpha}\left(w_{1}^{\alpha, \beta}\right)^{2}+\beta_{12}\left(w_{2}^{\alpha, \beta}\right)^{2}+2 a \mu_{1}\left(\varphi\left(\beta^{-\frac{1}{4}} y\right)\right)^{2}\right) \Phi \\
= & \left(\frac{e_{1}}{2}\left(d\left(y, \Omega_{\beta}^{*}\right)\right)^{2}-4 a^{2} e_{2} e_{3}\left(d\left(y, \Omega_{\beta}^{*}\right)\right)^{2}\right) \Phi \\
& +\left(\frac{e_{1}}{2}\left(d\left(y, \Omega_{\beta}^{*}\right)\right)^{2}-\frac{\beta_{11}}{\alpha}\left(w_{1}^{\alpha, \beta}\right)^{2}-\beta_{12}\left(w_{2}^{\alpha, \beta}\right)^{2}-2 a \mu_{1}\left(\varphi\left(\beta^{-\frac{1}{4}} y\right)\right)^{2}\right) \Phi .
\end{aligned}
$$

Copyright $\odot$ by SIAM. Unauthorized reproduction of this article is prohibited. 
In view of (3.58) and (3.61), we see that if $d\left(y, \partial \Omega_{\beta}^{*}\right) \geq m$ for large $m>0$,

$$
\frac{e_{1}}{2}\left(d\left(y, \Omega_{\beta}^{*}\right)\right)^{2}-\frac{\beta_{11}}{\alpha}\left(w_{1}^{\alpha, \beta}\right)^{2}-\beta_{12}\left(w_{2}^{\alpha, \beta}\right)^{2}-2 a \mu_{1}\left(\varphi\left(\beta^{-\frac{1}{4}} y\right)\right)^{2}>0 .
$$

We take small $a>0$ so that $\frac{e_{1}}{2}-4 a^{2} e_{2} e_{3} \geq \frac{e_{1}}{4}$. Then for $d\left(y, \partial \Omega_{\beta}^{*}\right) \geq m$,

$$
-\Delta \Phi+\left(\frac{\lambda_{1}}{\sqrt{\beta}}+\beta^{\frac{1}{2}} \beta_{13}\left(w_{3}^{\alpha, \beta}\left(\beta^{-\frac{1}{4}} y\right)\right)^{2}-\frac{\beta_{11}}{\alpha}\left(w_{1}^{\alpha, \beta}\right)^{2}-\beta_{12}\left(w_{2}^{\alpha, \beta}\right)^{2}\right) \Phi>0 .
$$

By (3.58), we also have $\lim _{R \rightarrow+\infty}\left\|v_{i, b}^{\alpha, \beta}\right\|_{L^{\infty}\left(\partial B_{R}(0)\right)}=0$ for $i=1,2$. Thus, it follows from the comparison principle that for some large $m>0$, independent of large $\alpha \# \beta^{1+\delta_{n}}$, there exists a constant $D>0$ such that

$$
w_{1}^{\alpha, \beta}(y) \leq D \Phi(y), \quad d\left(y, \partial \Omega_{\beta}^{*}\right) \geq m .
$$

Note that $e_{4} d(x, \partial \Omega) \leq \varphi(x), x \in \Omega$ for some $e_{4}>0$ and $\left\{w_{1}^{\alpha, \beta}, w_{2}^{\alpha, \beta}\right\}_{j}$ is bounded in $L^{\infty}\left(d\left(y, \partial \Omega_{\beta}^{*}\right) \leq m\right)$ by Proposition 9. Therefore, there exist constant $C, c>0$ such that

$$
w_{1}^{\alpha, \beta}(y) \leq C \exp \left(-c\left(d\left(y, \partial \Omega_{\beta}^{*}\right)^{2}\right)\right), \quad x \in \Omega_{\beta}^{*} .
$$

By the same argument with $w_{1}^{\alpha, \beta}$, we get the same estimate for $w_{2}^{\alpha, \beta}$. This implies the proof of (3.56) for $n=1,2$.

Second, we consider a case $n=3$. We recall from (3.31) that

$$
\Delta v_{1, c}^{\alpha, \beta}-\left(\frac{\lambda_{1}}{\beta}-\frac{\beta_{11}}{\alpha}\left(v_{1, c}^{\alpha, \beta}\right)^{2}-\beta_{12}\left(v_{2, c}^{\alpha, \beta}\right)^{2}+\beta_{13}\left(v_{3}^{\alpha, \beta}\left(\beta^{-\frac{1}{2}} y\right)\right)^{2}\right) v_{1, c}^{\alpha, \beta}=0 .
$$

Let

$$
\Psi(y)=\exp \left(-\sigma_{1}|y|\right)+\exp \left(-\frac{\sigma_{2}}{\sqrt{\beta}}\left(|y|-\frac{3 \sqrt{\beta}}{4} R_{0}\right)^{2}\right),
$$

where $\sigma_{1}, \sigma_{2}>0$ will be determined later. We have

$$
\begin{aligned}
& \Delta \Psi-\left(\frac{\lambda_{1}}{\beta}-\frac{\beta_{11}}{\alpha}\left(v_{1, c}^{\alpha, \beta}\right)^{2}-\beta_{12}\left(v_{2, c}^{\alpha, \beta}\right)^{2}+\beta_{13}\left(v_{3}^{\alpha, \beta}\left(\beta^{-\frac{1}{2}} y\right)\right)^{2}\right) \Psi \\
& =-\exp \left(-\sigma_{1}|y|\right) A(y)-\exp \left(-\frac{\sigma_{2}}{\sqrt{\beta}}\left(|y|-\frac{3 \sqrt{\beta}}{4} R_{0}\right)^{2}\right) B(y),
\end{aligned}
$$

where

$$
A(y)=\frac{\lambda_{1}}{\beta}-\frac{\beta_{11}}{\alpha}\left(v_{1, c}^{\alpha, \beta}\right)^{2}-\beta_{12}\left(v_{2, c}^{\alpha, \beta}\right)^{2}+\beta_{13}\left(v_{3}^{\alpha, \beta}\left(\beta^{-\frac{1}{2}} y\right)\right)^{2}-\sigma_{1}^{2}+\frac{2 \sigma_{1}}{|y|}
$$

and

$$
\begin{aligned}
B(y) & =\frac{\lambda_{1}}{\beta}-\frac{\beta_{11}}{\alpha}\left(v_{1, c}^{\alpha, \beta}\right)^{2}-\beta_{12}\left(v_{2, c}^{\alpha, \beta}\right)^{2}+\beta_{13}\left(v_{3}^{\alpha, \beta}\left(\beta^{-\frac{1}{2}} y\right)\right)^{2} \\
& -\frac{4 \sigma_{2}^{2}}{\beta}\left(|y|-\frac{3 \sqrt{\beta}}{4} R_{0}\right)^{2}+\frac{2 \sigma_{2}}{\sqrt{\beta}}+\frac{4 \sigma_{2}}{\sqrt{\beta}} \frac{\left(|y|-\frac{3 \sqrt{\beta}}{4} R_{0}\right)}{|y|} .
\end{aligned}
$$

Copyright $@$ by SIAM. Unauthorized reproduction of this article is prohibited. 
Fix a constant large $R>0$. We claim that

$$
A(y)>0 \text { and } B(y)>0 \text { if } R<|y| \leq \frac{3 R_{0} \sqrt{\beta}}{4} .
$$

Proposition 7 yields

$c_{0}\left(R_{0}-\beta^{-\frac{1}{2}}|y|\right) \leq v_{3}^{\alpha, \beta}\left(\beta^{-\frac{1}{2}} y\right) \leq c_{0}^{-1}\left(R_{0}-\beta^{-\frac{1}{2}}|y|\right)$ for some constant $0<c_{0}<1$.

By (3.68), (3.59), and (3.61), if $\sigma_{1}>0$ is sufficiently small and $R>0$ is large enough, then there is a constant $c_{1}, c_{2}>0$ satisfying

$$
\begin{aligned}
A(y) & \geq \frac{\lambda_{1}}{\beta}-\frac{\beta_{11}}{\alpha}\left(v_{1, c}^{\alpha, \beta}\right)^{2}-\beta_{12}\left(v_{2, c}^{\alpha, \beta}\right)^{2}+\beta_{13} c_{0}^{2}\left(R_{0}-\beta^{-\frac{1}{2}}|y|\right)^{2}-\sigma_{1}^{2}+\frac{2 \sigma_{1}}{|y|} \\
& \geq c_{1} R_{0}^{2}-\sigma_{1}^{2}+\frac{2 \sigma_{1}}{|y|}>c_{2}>0 \quad \text { if } \quad R<|y| \leq \frac{3 R_{0} \sqrt{\beta}}{4} .
\end{aligned}
$$

Moreover, we also see from (3.68), (3.59), and (3.61) that if $\sigma_{2}>0$ is sufficiently small and $R>0$ is large enough, then there is a constant $c_{3}>0$ such that

$$
\begin{aligned}
B(y) & \geq \frac{\lambda_{1}}{\beta}-\frac{\beta_{11}}{\alpha}\left(v_{1, c}^{\alpha, \beta}\right)^{2}-\beta_{12}\left(v_{2, c}^{\alpha, \beta}\right)^{2}+\beta_{13} c_{0}^{2}\left(R_{0}-\beta^{-\frac{1}{2}}|y|\right)^{2} \\
& -4 \sigma_{2}^{2}\left(\frac{3 R_{0}}{4}-\beta^{-\frac{1}{2}}|y|\right)^{2}-2 \sigma_{2}\left(\left(\frac{3 R_{0}}{4}-\beta^{-\frac{1}{2}}|y|\right)^{2}+|y|^{-2}\right) \\
& >c_{3}>0 \text { if } R<|y| \leq \frac{3 R_{0} \sqrt{\beta}}{4} .
\end{aligned}
$$

Now we see that the claim (3.67) holds. By the comparison principle, (3.59), and (3.61), there is a constant $c>0$ satisfying

$$
v_{1, c}^{\alpha, \beta}(y) \leq C \Psi(y) \text { for } R \leq|y| \leq \frac{3 \sqrt{\beta} R_{0}}{4}
$$

Let $\Upsilon(y)=\exp \left(-\frac{\sigma_{3}}{\sqrt{\beta}}\left(|y|-\sqrt{\beta} R_{0}\right)^{2}-\frac{\sigma_{3}}{\beta^{\frac{1}{4}}}\left(|y|-\frac{\sqrt{\beta} R_{0}}{2}\right)\right)$, where $\sigma_{3}>0$ will be determined later. Now we are going to show that there is a constant $C>0$ satisfying

$$
v_{1, c}^{\alpha, \beta}(y) \leq C \Upsilon(y) \text { for } \frac{\sqrt{\beta} R_{0}}{2} \leq|y| \leq \sqrt{\beta} R_{0} .
$$

We see that

$$
\Delta \Upsilon-\left(\frac{\lambda_{1}}{\beta}-\frac{\beta_{11}}{\alpha}\left(v_{1, c}^{\alpha, \beta}\right)^{2}-\beta_{12}\left(v_{2, c}^{\alpha, \beta}\right)^{2}+\beta_{13}\left(v_{3}^{\alpha, \beta}\left(\beta^{-\frac{1}{2}} y\right)\right)^{2}\right) \Upsilon(y)=-C(y) \Upsilon(y),
$$

where

$$
\begin{aligned}
C(y) & =\frac{\lambda_{1}}{\beta}-\frac{\beta_{11}}{\alpha}\left(v_{1, c}^{\alpha, \beta}\right)^{2}-\beta_{12}\left(v_{2, c}^{\alpha, \beta}\right)^{2}+\beta_{13}\left(v_{3}^{\alpha, \beta}\left(\beta^{-\frac{1}{2}} y\right)\right)^{2} \\
& -\left(\frac{2 \sigma_{3}}{\sqrt{\beta}}\left(|y|-\sqrt{\beta} R_{0}\right)+\frac{\sigma_{3}}{\beta^{\frac{1}{4}}}\right)^{2}+\frac{2 \sigma_{3}}{\sqrt{\beta}}+\frac{4 \sigma_{3}}{\sqrt{\beta}} \frac{\left(|y|-\sqrt{\beta} R_{0}\right)}{|y|}+\frac{2 \sigma_{3}}{\beta^{\frac{1}{4}}|y|} .
\end{aligned}
$$

Copyright $@$ by SIAM. Unauthorized reproduction of this article is prohibited. 
From (3.68) and (3.61), we see that if $\sigma_{3}>0$ is sufficiently small, then we see that as $\beta \rightarrow+\infty$,

$$
\begin{aligned}
C(y) & \geq \frac{\lambda_{1}}{\beta}-c_{4}^{2} \alpha^{-1} \beta^{-\frac{3}{4}}-c_{4}^{2} \beta^{-\frac{3}{4}}+\frac{2 \sigma_{3}}{\sqrt{\beta}}+\beta_{13} c_{0}^{2}\left(R_{0}-\beta^{-\frac{1}{2}}|y|\right)^{2} \\
& -2\left(4 \sigma_{3}^{2}\left(R_{0}-\beta^{-\frac{1}{2}}|y|\right)^{2}+\frac{\sigma_{3}^{2}}{\sqrt{\beta}}\right)-2 \sigma_{3}\left(\left(R_{0}-\beta^{-\frac{1}{2}}|y|\right)^{2}+|y|^{-2}\right) \\
& >0 \quad \text { for } \quad \frac{R_{0} \sqrt{\beta}}{2} \leq|y| \leq R_{0} \sqrt{\beta} .
\end{aligned}
$$

If $0<\sigma_{3} \ll \sigma_{i} \ll 1, i=1,2$, then the comparison principle, (3.71), and $\left.v_{1, c}^{\alpha, \beta}(y)\right|_{|y|=\sqrt{\beta} R_{0}}$ $=0$ implies that there is a constant $C>0$ such that if $\frac{\sqrt{\beta} R_{0}}{2} \leq|y| \leq \sqrt{\beta} R_{0}$, then

$$
v_{1, c}^{\alpha, \beta}(y) \leq C \Upsilon(y)=C \exp \left(-\frac{\sigma_{3}}{\sqrt{\beta}}\left(|y|-\sqrt{\beta} R_{0}\right)^{2}-\frac{\sigma_{3}}{\beta^{\frac{1}{4}}}\left(|y|-\frac{\sqrt{\beta} R_{0}}{2}\right)\right) .
$$

The above estimation also holds for $v_{2, c}^{\alpha, \beta}$. In view of (3.75), we see that

$$
\sum_{i=1}^{2} v_{i, c}^{\alpha, \beta} \leq\left\{\begin{array}{l}
C \exp \left(-\frac{\sigma_{3}}{\sqrt{\beta}}\left(|y|-\sqrt{\beta} R_{0}\right)^{2}\right) \quad \text { if } \frac{\sqrt{\beta} R_{0}}{2} \leq|y| \leq \frac{3 \sqrt{\beta} R_{0}}{4} \\
C \exp \left(-\frac{\sigma_{3} R_{0} \beta^{\frac{1}{4}}}{4}\right) \quad \text { if } \quad \frac{3 \sqrt{\beta} R_{0}}{4} \leq|y| \leq \sqrt{\beta} R_{0} .
\end{array}\right.
$$

Let $\Phi(y)=\exp \left(-\sigma_{4} \sqrt{\beta}\right)$, where $\sigma_{4}>0$ will be determined later. Now we are going to show that there is a constant $C>0$ satisfying

$$
v_{1, c}^{\alpha, \beta}(y) \leq C \Phi(y) \text { for } \frac{3 \sqrt{\beta} R_{0}}{4} \leq|y| \leq \sqrt{\beta} R_{0} .
$$

We see that

$$
\Delta \Phi-\left(\frac{\lambda_{1}}{\beta}-\frac{\beta_{11}}{\alpha}\left(v_{1, c}^{\alpha, \beta}\right)^{2}-\beta_{12}\left(v_{2, c}^{\alpha, \beta}\right)^{2}+\beta_{13}\left(v_{3}^{\alpha, \beta}\left(\beta^{-\frac{1}{2}} y\right)\right)^{2}\right) \Phi(y)=-D(y) \Phi(y),
$$

where

$$
D(y)=\frac{\lambda_{1}}{\beta}-\frac{\beta_{11}}{\alpha}\left(v_{1, c}^{\alpha, \beta}\right)^{2}-\beta_{12}\left(v_{2, c}^{\alpha, \beta}\right)^{2}+\beta_{13}\left(v_{3}^{\alpha, \beta}\left(\beta^{-\frac{1}{2}} y\right)\right)^{2} .
$$

From (3.76), we see that as $\beta \rightarrow+\infty$,

$$
\begin{aligned}
D(y) & \geq \frac{\lambda_{1}}{\beta}-c \exp \left(-\frac{\sigma_{3} R_{0} \beta^{\frac{1}{4}}}{2}\right)+\beta_{13} c_{0}^{2}\left(R_{0}-\beta^{-\frac{1}{2}}|y|\right)^{2} \\
& >0 \quad \text { for } \quad \frac{3 R_{0} \sqrt{\beta}}{4} \leq|y| \leq R_{0} \sqrt{\beta} .
\end{aligned}
$$

If $0<\sigma_{4} \ll \sigma_{i} \ll 1, i=1,2,3$, then the comparison principle, (3.76), and $\left.v_{1, c}^{\alpha, \beta}(y)\right|_{|y|=\sqrt{\beta} R_{0}}$ $=0$ implies that there is a constant $C>0$ such that if $\frac{3 \sqrt{\beta} R_{0}}{4} \leq|y| \leq \sqrt{\beta} R_{0}$, then

$$
v_{1, c}^{\alpha, \beta}(y) \leq C \Phi(y)=C \exp \left(-\sigma_{4} \sqrt{\beta}\right) .
$$

Together with (3.71) and (3.75), by choosing small positive constants $0<c \ll$ $\sigma_{4} \ll \sigma_{i} \ll 1, i=1,2,3$, we see that (3.56) also holds for $n=3$.

Copyright (c) by SIAM. Unauthorized reproduction of this article is prohibited. 
Now, we prove the following convergence result for the first and second components.

Proposition 12. (i) If $n=1,2$, there is a positive least energy solution $\left(v_{1, b}, v_{2, b}\right)$ of (1.5) such that $\left(v_{1, b}^{\alpha, \beta}, v_{2, b}^{\alpha, \beta}\right) \rightarrow\left(v_{1, b}, v_{2, b}\right)$ in $\left[H^{1}\left(\Omega_{b}^{\beta}\right)\right]^{2}$, up to a subsequence, as $\alpha \# \beta^{1+\delta_{n}} \rightarrow \infty$.

(ii) If $n=3$, there is a positive least energy solution $\left(v_{1, c}, v_{2, c}\right)$ of (1.11) such that $\left(v_{1, c}^{\alpha, \beta}, v_{2, c}^{\alpha, \beta}\right) \rightarrow\left(v_{1, c}, v_{2, c}\right)$ in $\left[H^{1}\left(\Omega_{c}^{\beta}\right)\right]^{2}$, up to a subsequence, as $\alpha \# \beta^{1+\delta_{n}} \rightarrow \infty$.

Proof. In view of Propositions 5 and 8 , it holds that for $n=1,2$,

$$
\begin{aligned}
& \lim _{\alpha \# \beta^{1+\delta_{n}} \rightarrow \infty} 2 \beta_{12}\left|v_{1, b}^{\alpha, \beta} v_{2, b}^{\alpha, \beta}\left(R_{0}-\beta^{-\frac{1}{4}}|y|\right)^{\frac{n-1}{2}}\right|_{2, \Omega_{b}^{\beta}}^{2}\left|S^{n-1}\right| \\
& =\lim _{\alpha \# \beta^{1+\delta_{n}} \rightarrow \infty}\left[\left(\left|\nabla v_{1, b}^{\alpha, \beta}\left(R_{0}-\beta^{-\frac{1}{4}}|y|\right)^{\frac{n-1}{2}}\right|_{2, \Omega_{b}^{\beta}}^{2}+\left|\nabla v_{2, b}^{\alpha, \beta}\left(R_{0}-\beta^{-\frac{1}{4}}|y|\right)^{\frac{n-1}{2}}\right|_{2, \Omega_{b}^{\beta}}^{2}\right.\right. \\
& \left.\left.+\sum_{i=1}^{2} \beta_{i 3} \int_{\Omega_{b}^{\beta}} \sqrt{\beta}\left(v_{3}^{\alpha, \beta}\left(R_{0}-\beta^{-\frac{1}{4}}|y|\right)\right)^{2}\left(v_{i, b}^{\alpha, \beta}\right)^{2}\left(R_{0}-\beta^{-\frac{1}{4}}|y|\right)\right)^{n-1} d y\right)\left|S^{n-1}\right| \\
& \left.+\beta^{\frac{5}{4}-\frac{n}{2}}\left\{\sum_{i=1}^{2}\left(\left|\nabla v_{i, c}^{\alpha, \beta}\right|_{2, \Omega_{c}^{\beta}}^{2}+\frac{\lambda_{i}}{\beta}\left|v_{i, c}^{\alpha, \beta}\right|_{2, \Omega_{c}^{\beta}}^{2}+\beta_{i 3} \int_{\Omega_{c}^{\beta}}\left(v_{3}^{\alpha, \beta}\left(\beta^{-\frac{1}{2}} y\right)\right)^{2}\left(v_{i, c}^{\alpha, \beta}\right)^{2} d y\right)\right\}\right] \\
& \leq 4 M_{b}\left|S^{n-1}\right| R_{0}^{n-1},
\end{aligned}
$$

and for $n=3$,

$$
\begin{aligned}
& \lim _{\alpha \# \beta^{1+\delta_{n}} \rightarrow \infty} 2 \beta_{12}\left|v_{1, c}^{\alpha, \beta} v_{2, c}^{\alpha, \beta}\right|_{2, \Omega_{c}^{\beta}}^{2} \\
& =\lim _{\alpha \# \beta^{1+\delta_{n}} \rightarrow \infty}\left[\left(\left|\nabla v_{1, c}^{\alpha, \beta}\right|_{2, \Omega_{c}^{\beta}}^{2}+\left|\nabla v_{2, c}^{\alpha, \beta}\right|_{2, \Omega_{c}^{\beta}}^{2}+\sum_{i=1}^{2} \beta_{i 3} \int_{\Omega_{c}^{\beta}}\left(v_{3}^{\alpha, \beta}\left(\beta^{-\frac{1}{2}} y\right)\right)^{2}\left(v_{i, c}^{\alpha, \beta}\right)^{2} d y\right)\right. \\
& +\beta^{\frac{n}{2}-\frac{5}{4}}\left|S^{n-1}\right|\left\{\sum_{i=1}^{2}\left(\left|\nabla v_{i, b}^{\alpha, \beta}\left(R_{0}-\beta^{-\frac{1}{4}}|y|\right)^{\frac{n-1}{2}}\right|_{2, \Omega_{b}^{\beta}}^{2}+\frac{\lambda_{i}}{\sqrt{\beta}}\left|v_{i, b}^{\alpha, \beta}\left(R_{0}-\beta^{-\frac{1}{4}}|y|\right)^{\frac{n-1}{2}}\right|_{2, \Omega_{b}^{\beta}}^{2}\right)\right. \\
& \left.\left.+\sum_{i=1}^{2} \beta_{i 3} \int_{\Omega_{b}^{\beta}} \sqrt{\beta}\left(v_{3}^{\alpha, \beta}\left(R_{0}-\beta^{-\frac{1}{4}}|y|\right)\right)^{2}\left(v_{i, b}^{\alpha, \beta}\right)^{2}\left(R_{0}-\beta^{-\frac{1}{4}}|y|\right)^{n-1} d y\right\}\right] \\
& \leq 4 M_{c} .
\end{aligned}
$$

We define

$$
\breve{v}^{\alpha, \beta}(y)=\left(v_{1}^{\alpha, \beta}(y), v_{2}^{\alpha, \beta}(y)\right)=\left\{\begin{array}{l}
\left(v_{1, b}^{\alpha, \beta}(y), v_{2, b}^{\alpha, \beta}(y)\right) \text { if } n=1,2 \\
\left(v_{1, c}^{\alpha, \beta}(y), v_{2, c}^{\alpha, \beta}(y)\right) \text { if } n=3
\end{array}\right.
$$

and

$$
\Omega^{\beta}=\left\{\begin{array}{l}
\Omega_{b}^{\beta} \text { if } n=1,2 \\
\Omega_{c}^{\beta} \text { if } n=3
\end{array}\right.
$$

From Proposition 8, we see that $\breve{v}^{\alpha, \beta}$ is uniformly bounded in $\left[H^{1}\left(\Omega^{\beta}\right)\right]^{2}$. Thus we can choose a subsequence $\alpha_{j}, \beta_{j}$ of $\alpha, \beta$ with $\lim _{j \rightarrow \infty} \alpha_{j} \# \beta_{j}^{1+\delta_{n}}=\infty$ such that there is $\breve{v}=\left(v_{1}, v_{2}\right)$ satisfying $\breve{v}^{j} \equiv \breve{v}^{\alpha_{j}, \beta_{j}} \rightarrow \breve{v}$ weakly in $\left[H^{1}\left(\Omega^{\beta_{j}}\right)\right]^{2}$ and strongly in

Copyright (c) by SIAM. Unauthorized reproduction of this article is prohibited. 
$\left[L_{\text {loc }}^{4}\left(\Omega^{\beta_{j}}\right)\right]^{2}$ as $j \rightarrow \infty$. From Propositions 3 and 7 , we recall that $u_{3}^{\alpha_{j}, \beta_{j}}=v_{3}^{\alpha_{j}, \beta_{j}} \rightarrow U_{3}$ in $C^{1}(\overline{\{x \in \Omega|| x \mid \geq R\}})$ for $n=1,2$ and in $C(\Omega)$ for $n=3$ as $j \rightarrow \infty$ where $U_{3}$ is a least energy solution of (1.4). Then by using test functions, it is easy to see that $\breve{v}$ is a weak solution of (1.5) for $n=1,2$ and (1.11) for $n=3$. In view of Proposition 10, we have $v_{1} \neq 0$ and $v_{2} \neq 0$. By (3.81) and (3.82), we get that if $n=1,2$,

$$
\begin{aligned}
& 4 M_{b}\left|S^{n-1}\right| R_{0}^{n-1} \\
& \leq \sum_{i=1}^{2}\left(\left|\frac{d v_{i}}{d t}\right|_{2, \mathbb{R}_{+}^{1}}^{2}+\beta_{i 3} \int_{\mathbb{R}_{+}^{1}}\left(\frac{d U_{3}\left(R_{0}\right)}{d r}\right)^{2} v_{i}^{2} t^{2} d t\right)\left|S^{n-1}\right| R_{0}^{n-1} \\
& \leq \lim _{j \rightarrow \infty} \sum_{i=1}^{2}\left(\left|\nabla v_{i, b}^{\alpha_{j}, \beta_{j}}\left(R_{0}-\beta_{j}^{-\frac{1}{4}}|y|\right)^{\frac{n-1}{2}}\right|_{2, \Omega_{b}^{\beta_{j}}}^{2}\right. \\
& \left.\left.+\beta_{i 3} \int_{\Omega_{b}^{\beta_{j}}} \sqrt{\beta_{j}}\left(v_{3}^{\alpha_{j}, \beta_{j}}\left(R_{0}-\beta_{j}^{-\frac{1}{4}}|y|\right)\right)^{2}\left(v_{i, b}^{\alpha_{j}, \beta_{j}}\right)^{2}\left(R_{0}-\beta_{j}^{-\frac{1}{4}}|y|\right)\right)^{n-1} d y\right)\left|S^{n-1}\right| \\
& \leq 4 M_{b}\left|S^{n-1}\right| R_{0}^{n-1},
\end{aligned}
$$

and if $n=3$,

$$
\begin{aligned}
4 M_{c} & \leq \sum_{i=1}^{2}\left(\left|\nabla v_{i}\right|_{2, \mathbb{R}^{n}}^{2}+\beta_{i 3} \int_{\mathbb{R}^{n}}\left(U_{3}(0)\right)^{2}\left(v_{i}\right)^{2} d y\right) \\
& \leq \lim _{j \rightarrow \infty} \sum_{i=1}^{2}\left(\left|\nabla v_{i, c}^{\alpha_{j}, \beta_{j}}\right|_{2, \Omega_{c}^{\beta_{j}}}^{2}+\beta_{i 3} \int_{\Omega_{c}^{\beta_{j}}}\left(v_{3}^{\alpha_{j}, \beta_{j}}\left(\beta_{j}^{-\frac{1}{2}} y\right)\right)^{2}\left(v_{i, c}^{\alpha_{j}, \beta_{j}}\right)^{2} d y\right) \\
& \leq 4 M_{c} .
\end{aligned}
$$

Together with the exponential decay of $\breve{v}^{j}$ in Proposition 11, we note that not only does $\breve{v}^{j}$ weekly converge to $\breve{v}$ in $\left[H^{1}\left(\Omega^{\beta_{j}}\right)\right]^{2}$, but also the $\left[H^{1}\left(\Omega^{\beta_{j}}\right)\right]^{2}$-norm of $\breve{v}^{j}$ converges to the $\left[H^{1}\left(\Omega^{\beta_{j}}\right)\right]^{2}$-norm of $\breve{v}$. Therefore, $\breve{v}^{j}$ strongly converges to $\breve{v}$ in $\left[H^{1}\left(\Omega^{\beta_{j}}\right)\right]^{2}$. Moreover, (3.85) and (3.86) imply $\breve{v}$ is a positive least energy solution of (1.5) for $n=1,2$ and (1.11) for $n=3$. Now we complete the proof of Proposition 12.

3.4. Proof of Theorem 1. Completion of Proof for Theorem 1. In the preceding propositions, we have proved all results in Theorem 1 except (1.17). We recall the notations $K$ and $G$ in (3.14) and (3.15). In view of Proposition 3 and Lemma 2(ii), we see that

$$
\begin{aligned}
c_{\alpha, \beta} & =I\left(\vec{u}^{\alpha, \beta}\right)=\frac{\left\|u_{3}^{\alpha, \beta}\right\|_{\lambda_{3}, \Omega}^{4}}{4 \beta_{33}\left|u_{3}^{\alpha, \beta}\right|_{4, \Omega}^{4}}+\frac{K\left(\vec{u}^{\alpha, \beta}\right)}{4 \beta_{33}\left|u_{3}^{\alpha, \beta}\right|_{4, \Omega}^{4} G\left(\vec{u}^{\alpha, \beta}\right)} \\
& \geq L+\frac{K\left(\vec{u}^{\alpha, \beta}\right)}{4 \beta_{33}\left|u_{3}^{\alpha, \beta}\right|_{4, \Omega}^{4} G\left(\vec{u}^{\alpha, \beta}\right)},
\end{aligned}
$$

here we used $L=\inf _{u \in H_{0}^{1}(\Omega)} \frac{\|u\|_{\lambda_{3}, \Omega}^{4}}{4 \beta_{33}|u|_{4, \Omega}^{4}}$. By (3.3) and Proposition 3(iii), we get $(3.88)$

$I\left(\vec{u}^{\alpha, \beta}\right)$

$\geq L+\frac{(L+o(1))\left(\left\|u_{1}^{\alpha, \beta}\right\|_{\lambda_{1}, \Omega}^{2}+\left\|u_{2}^{\alpha, \beta}\right\|_{\lambda_{2}, \Omega}^{2}+\beta \beta_{13}\left|u_{1}^{\alpha, \beta} u_{3}^{\alpha, \beta}\right|_{2, \Omega}^{2}+\beta \beta_{23}\left|u_{2}^{\alpha, \beta} u_{3}^{\alpha, \beta}\right|_{2, \Omega}^{2}\right)^{2}}{G\left(\vec{u}^{\alpha, \beta}\right)}$.

Copyright $@$ by SIAM. Unauthorized reproduction of this article is prohibited. 
From Proposition 3(iii), we also note that

$$
\begin{aligned}
G\left(\vec{u}^{\alpha, \beta}\right) & =\left(\beta_{11}\left|u_{1}^{\alpha, \beta}\right|_{4, \Omega}^{4}+\beta_{22}\left|u_{2}^{\alpha, \beta}\right|_{4, \Omega}^{4}+2 \alpha \beta_{12}\left|u_{1}^{\alpha, \beta} u_{2}^{\alpha, \beta}\right|_{2, \Omega}^{2}\right) \beta_{33}\left|u_{3}^{\alpha, \beta}\right|_{4, \Omega}^{4} \\
& -\left(\beta \beta_{13}\left|u_{1}^{\alpha, \beta} u_{3}^{\alpha, \beta}\right|_{2, \Omega}^{2}+\beta \beta_{23}\left|u_{2}^{\alpha, \beta} u_{3}^{\alpha, \beta}\right|_{2, \Omega}^{2}\right)^{2} \\
& =\left(\beta_{11}\left|u_{1}^{\alpha, \beta}\right|_{4, \Omega}^{4}+\beta_{22}\left|u_{2}^{\alpha, \beta}\right|_{4, \Omega}^{4}+2 \alpha \beta_{12}\left|u_{1}^{\alpha, \beta} u_{2}^{\alpha, \beta}\right|_{2, \Omega}^{2}\right)(4 L+o(1)) \\
& -\left(\beta \beta_{13}\left|u_{1}^{\alpha, \beta} u_{3}^{\alpha, \beta}\right|_{2, \Omega}^{2}+\beta \beta_{23}\left|u_{2}^{\alpha, \beta} u_{3}^{\alpha, \beta}\right|_{2, \Omega}^{2}\right)^{2} .
\end{aligned}
$$

Using the exponential decay and the convergence in Propositions 11 and 12 for the scaling of $u_{i}^{\alpha, \beta}$ depending on the space dimension $n$, we get by the same argument with Proposition 4 that

$$
\begin{aligned}
c_{\alpha, \beta} \geq \min \left\{L+\frac{\beta^{\frac{3}{4}}}{\alpha}\left(M_{b}\left|S^{n-1}\right| R_{0}^{n-1}+o(1)\right), L+\frac{\beta^{2-\frac{n}{2}}}{\alpha}\left(M_{c}+o(1)\right)\right\} \\
=\left\{\begin{array}{l}
L+\frac{\beta^{\frac{3}{4}}}{\alpha}\left(M_{b}\left|S^{n-1}\right| R_{0}^{n-1}+o(1)\right) \text { as } \alpha \# \beta^{\frac{3}{4}} \rightarrow \infty \text { if } n=1,2, \\
L+\frac{\beta^{\frac{1}{2}}}{\alpha}\left(M_{c}+o(1)\right) \text { as } \alpha \# \beta^{\frac{1}{2}} \rightarrow \infty \text { if } n=3 .
\end{array}\right.
\end{aligned}
$$

Since we have already the opposite estimate in Proposition 4, we complete the proof of Theorem 1.

Acknowledgment. The authors express their sincere gratitude to anonymous referees for their careful reading and helpful comments.

\section{REFERENCES}

[1] A. Ambrosetti And E. Colorado, Standing waves of some coupled nonlinear Schrödinger equations, J. Lond. Math. Soc. (2), 75 (2007), pp. 67-82.

[2] T. Bartsch, E. N. Dancer, And Z.-Q. WAng, A Liouville theorem, a-priori bounds, and bifurcating branches of positive solutions for a nonlinear elliptic system, Calc. Var. Partial Differential Equations, 37 (2010), pp. 345-361.

[3] T. BARTsch And Z.-Q. WANG, Note on ground states of nonlinear Schrödinger systems, J. Partial Differential Equations, 19 (2006), pp. 200-207.

[4] T. Bartsch, Z.-Q. Wang, and J. Wei, Bound states for a coupled Schrödinger system, J. Fixed Point Theory Appl., (2007), pp. 353-367.

[5] J. BYEOn, Existence of large positive solutions of some nonlinear elliptic equations on singularly perturbed domains, Comm. Partial Differential Equations, 22 (1997), pp. 1731-1769.

[6] J. Byeonz, Semi-classical standing waves for nonlinear Schrödinger systemsz, Calc. Var. Partial Differential Equations, 54 (2015), pp. 2287-2340.

[7] J. Byeon, Y. SAto, And Z.-Q. Wang, Pattern formation via mixed attractive and repulsive interactions for nonlinear Schrödinger systems, J. Math. Pures Appl. (9), 106 (2016), pp. $477-511$.

[8] J. Byeon, Y. SAto, And Z.-Q. WANG, Pattern formation via mixed interactions for coupled Schrödinger equations under Neumann boundary condition, J. Fixed Point Theory Appl., 19 (2017), pp. 559-583.

[9] S. Chang, C. S. Lin, T. C. Lin, And W. Lin, Segregated nodal domains of two-dimensional multispecies Bose-Einstein condensates, Phys. D, 196 (2004), pp. 341-361.

[10] M. Conti, S. Terracini, and G. Verzini, Nehari's problem and competing species system, Ann. Inst. H. Poincaré Anal. Non Linéaire, 19 (2002), pp. 871-888.

[11] E. N. Dancer, J. Wei, And T. Weth, A priori bounds versus multiple existence of positive solutions for a nonlinear Schrödinger system, Ann. Inst. H. Poincaré Anal. Non Linéaire, 27 (2010), pp. 953-969.

[12] B. Gidas, W.-M. Ni, And L. NiRenberg, Symmetry and related properties via the maximum principle, Comm. Math. Phys., 68 (1979), pp. 209-243.

[13] D. Gilbarg and N. S. Trudinger, Elliptic Partial Differential Equations of Second Order, 2nd ed., Grundlehren der Mathematischen Wissenschaften 224, Springer-Verlag, Berlin, 1983.

Copyright $@$ by SIAM. Unauthorized reproduction of this article is prohibited. 
[14] N. IKoma AND K. TANAKA, A local mountain pass type result for a system of nonlinear Schrödinger equations, Calc. Var. Partial Differential Equations, 40 (2011), pp. 449-480.

[15] M.K. Kwong, Uniqueness of positive solutions of $\Delta u+f(u)=0$ in $\mathbb{R}^{n}$, Arch. Ration. Mech. Anal., 105 (1989), pp. 243-266.

[16] T.-C. Lin AND J. WeI, Ground state of $N$ coupled nonlinear Schrödinger equations in $R^{n}, n \leq$ 3, Comm. Math. Phys., 255 (2005), pp. 629-653.

[17] T.-C. Lin And J. WeI, Spikes in two coupled nonlinear Schrödinger equations, Ann. Inst. H. Poincaré Anal. Non Linéaire, 22 (2005), pp. 403-439.

[18] P. L. Lions, The concentration-compactness principle in the calculus of variations, The locally compact case, II, Ann. Inst. H. Poincaré Anal. Non Linéaire, 1 (1984), pp. 223-283.

[19] J. LiU, X. LiU, AND Z.-Q. WANG, Multiple mixed states of nodal solutions for nonlinear Schrödinger systems, Calc. Var. Partial Differential Equations, 52 (2015), pp. 565-586.

[20] Z. L. LiU AND Z.-Q. WANG, Multiple bound states of nonlinear Schrödinger systems, Comm. Math. Phys., 282 (2008), pp. 721-731.

[21] Z. L. LIU AND Z.-Q. WANG, Ground states and bound states of a nonlinear Schrödinger system, Adv. Nonlinear Stud., 10 (2010), pp. 175-193.

[22] L.A. Maia, E. Montefusco, And B. Pellacci, Positive solutions for a weakly coupled nonlinear Schrödinger system, J. Differential Equations, 299 (2006), pp. 743-767.

[23] M. Mitchell And M. Segev, Self-trapping of inconherent white light, Nature, 387 (1997), pp. $880-882$.

[24] E. Montefusco, B. Pellacci, and M. Squassina, Semiclassical states for weakly coupled nonlinear Schrödinger systems, J. Eur. Math. Soc., 10 (2008), pp. 41-71.

[25] B. Noris AND M. RAmos, Existence and bounds of positive solutions for a nonlinear Schrödinger system, Proc. Amer. Math. Soc., 138 (2010), pp. 1681-1692.

[26] B. Noris, H. Tavares, S. Terracini, and G. Verzini, Uniform Hölder bounds for nonlinear Schrödinger systems with strong competition, Comm. Pure and Appl. Math., 63 (2010), pp. 267-302.

[27] S. PEng AND Z.-Q. WANG, Segregated and synchronized vector solutions for nonlinear Schrödinger systems, Arch. Ration. Mech. Anal., 208 (2013), pp. 305-339.

[28] Ch. RÜEGG ET AL., Bose-Einstein condensation of the triple states in the magnetic insulator $\mathrm{TlCuCl}_{3}$, Nature, 423 (2003), pp. 62-65.

[29] Y. SAто, Multi-peak Solutions for Nonlinear Schrödinger Equations, thesis, Waseda University, Tokyo, Japan, 2007.

[30] Y. SATO AND Z.-Q. WANG, On the multiple existence of semi-positive solutions for a nonlinear Schrödinger system, Ann. Inst. H. Poincaré Anal. Non Linéaire, 30 (2013), pp. 1-22.

[31] Y. SATO AND Z.-Q. WANG, On the least energy sign-changing solutions for a nonlinear elliptic system, Discrete Contin. Dyn. Syst., 35 (2015), pp. 2151-2164.

[32] Y. SATO AND Z.-Q. WANG, Least energy solutions for nonlinear Schrödinger systems with mixed attractive and repulsive couplings, Adv. Nonlinear Stud., 15 (2015), pp. 1-22.

[33] Y. SATO AND Z.-Q. WANG, Multiple positive solutions for Schrödinger systems with mixed couplings, Calc. Var. Partial Differential Equations, 54 (2015), pp. 1373-1392.

[34] B. Sirakov, Least energy solitary waves for a system of nonlinear Schrödinger equations in $R^{n}$, Comm. Math. Phys., 271 (2007), pp. 199-221.

[35] N. SOAVE, On existence and phase separation of solitary waves for nonlinear Schrödinger systems modelling simultaneous cooperation and competition, Calc. Var. Partial Differential Equations, 53 (2015), pp. 689-718.

[36] N. SoAVE AND H. TAVARES, New existence and symmetry results for least energy positive solutions of Schrödinger systems with mixed competition and cooperation terms, J. Differential Equations, 261 (2016), pp. 505-537.

[37] H. Tavares and S. Terracini, Sign-changing solutions of competition-diffusion elliptic systems and optimal partition problems, Ann. Inst. H. Poincaré Anal. Non Linéaire, 29 (2012), pp. 279-300.

[38] H. Tavares, S. Terracini, G. Verzini, and T. Weth, Existence and nonexistence of entire solutions for non-cooperative cubic elliptic systems, Comm. Partial Differential Equations, 36 (2011), pp. 1988-2010.

[39] S. Terracini and G. Verzini, Multipulse Phase in k-mixtures of Bose-Einstein condensates, Arch. Rat. Mech. Anal., 194 (2009), pp. 717-741.

[40] R. TIAn AND Z.-Q. WANG, Multiple solitary wave solutions of nonlinear Schrödinger systems, Topol. Methods Nonlinear Anal., 37 (2011), pp. 203-223.

[41] Z.-Q. Wang AND M. Willem, Partial symmetry of vector solutions for elliptic systems, J. Anal. Math., 122 (2014), pp. 69-85.

Copyright $@$ ( ) by SIAM. Unauthorized reproduction of this article is prohibited. 
[42] J. Wei AND T. Weth, Nonradial symmetric bound states for a system of two coupled Schrödinger equations, Rend. Lincei Mat. Appl., 18 (2007), pp. 279-293.

[43] J. Wei AND T. Weth, Radial solutions and phase separation in a system of two coupled Schrödinger equations, Arch. Ration. Mech. Anal., 190 (2008), pp. 83-106.

[44] J. Wei AND W. YAO, Uniqueness of positive solutions to some coupled nonlinear Schrödinger equations, Commun. Pure Appl. Anal., 11 (2012), pp. 1003-1011.

Copyright $@$ by SIAM. Unauthorized reproduction of this article is prohibited. 